\title{
Working memory related brain network connectivity in individuals with schizophrenia and their siblings
}

\author{
Grega Repovš ${ }^{1}$ and Deanna M. Barch ${ }^{2,3,4}$ \\ ${ }^{1}$ Department of Psychology, University of Ljubljana, Ljubljana, Slovenia \\ ${ }^{2}$ Department of Psychology, Washington University in St. Louis, St. Louis, MO, USA \\ ${ }^{3}$ Department of Psychiatry, Washington University in St. Louis, St. Louis, MO, USA \\ ${ }^{4}$ Department of Radiology, Washington University in St. Louis, St. Louis, MO, USA
}

\section{Edited by:}

Vince D. Calhoun, University of New

Mexico, USA

Reviewed by:

Stuart R. Steinhauer, University of Pittsburgh, USA

Stefan Ehrlich, University Hospital

Carl Gustav Carus, Germany

Scott R. Sponheim, Minneapolis

Veterans Affairs Medical Center, USA

${ }^{*}$ Correspondence:

Grega Repovš, Department of

Psychology, University of Ljubljana,

Askerceva 2, SI-1000 Ljubljana,

Slovenia.

e-mail: grega.repovs@psy.ff.uni-lj.si
A growing number of studies have reported altered functional connectivity in schizophrenia during putatively "task-free" states and during the performance of cognitive tasks. However, there have been few systematic examinations of functional connectivity in schizophrenia across rest and different task states to assess the degree to which altered functional connectivity reflects a stable characteristic or whether connectivity changes vary as a function of task demands. We assessed functional connectivity during rest and during three working memory loads of an $N$-back task (0-back, 1-back, 2-back) among: (1) individuals with schizophrenia $(N=19) ;(2)$ the siblings of individuals with schizophrenia $(N=28)$; (3) healthy controls $(N=10)$; and (4) the siblings of healthy controls ( $N=17)$. We examined connectivity within and between four brain networks: (1) frontal-parietal (FP); (2) cingulo-opercular (CO); (3) cerebellar (CER); and (4) default mode (DMN). In terms of within-network connectivity, we found that connectivity within the DMN and FP increased significantly between resting state and 0-back, while connectivity within the CO and CER decreased significantly between resting state and 0-back. Additionally, we found that connectivity within both the DMN and FP was further modulated by memory load. In terms of between network connectivity, we found that the DMN became significantly more "anticorrelated" with the FP, CO, and CER networks during 0-back as compared to rest, and that connectivity between the FP and both $\mathrm{CO}$ and CER networks increased with memory load. Individuals with schizophrenia and their siblings showed consistent reductions in connectivity between both the FP and CO networks with the CER network, a finding that was similar in magnitude across rest and all levels of working memory load. These findings are consistent with the hypothesis that altered functional connectivity in schizophrenia reflects a stable characteristic that is present across cognitive states.

Keywords: schizophrenia, functional connectivity, working memory, cognitive control, cerebellum, task, risk

\section{INTRODUCTION}

A growing body of work focused on understanding the neurobiological bases of cognitive impairment in schizophrenia suggests that changes in the function of a single brain region, or even a brain system, cannot explain the functional impairments seen in this illness. Instead, research has increasingly focused on understanding the integrity of neural circuits that work together to support sensory, cognitive, and emotional processes (Calhoun et al., 2009). This approach to understanding schizophrenia is consistent with the hypothesis that this illness reflects a "dysconnection" syndrome (Stephan et al., 2009). Much of this work has focused on examining different aspects of functional brain connectivity, either when the individual is at rest, or when the individual is performing a specific task (e.g., working memory). Both types of studies have provided robust evidence for altered functional connectivity in schizophrenia (Brown and Thompson, 2010). However, few studies have examined functional connectivity in schizophrenia across both resting and active task states. As such, it is difficult to know to what degree such impairments are state dependent or reflective of more fundamental and stable changes in brain organization in schizophrenia. Thus, the goal of the current study was to examine functional brain connectivity in known neural networks during rest and during different working memory loads in individuals with schizophrenia, their siblings, and healthy controls.

Functional brain connectivity is an approach to understanding brain function that examines the covariance in activity across brain regions. One common approach to assessing connectivity is to use blood oxygen level dependent (BOLD) timeseries acquired using fMRI (often referred to as fcMRI), either while the person is resting or while the person is engaged in a particular task. FcMRI data can be analyzed in a variety of ways, including hypothesis driven approaches that start with the identification of one or more regions of interest (ROIs) and either examine the covariance of a timeseries from this region with all other voxels in the brain or with the timeseries from specific other ROIs. Alternatively, one can use a more data driven approach, such as independent components analysis, that identifies groups of brain regions showing covarying timeseries at differing spatial scales. One hypothesis about the 
meaning of fcMRI is that it identifies brain regions that have a history of "working together" and that likely reflect a combination of both structural connectivity and more indirect connections (Fair et al., 2007a,b,c; Dosenbach et al., 2010). It is conceptually similar to other measures of assessing coordinated activity across the brain, such as EEG coherence, but differs in the time scale (on the order of seconds for fcMRI versus milliseconds for EEG coherence) and spatial resolution (higher for fcMRI than EEG coherence).

As noted above, numerous studies have now examined functional brain connectivity in schizophrenia during rest states. Several of these studies have examined characteristics of functional brain connectivity using graph theoretic approaches. These studies have found evidence for altered "small-world" network characteristics in schizophrenia, including reduced efficiency, increased path lengths, and reduced clustering coefficients (Bassett et al., 2008; Liu et al., 2008; Lynall et al., 2010; Yu et al., 2011). Other work has provided evidence for reduced global brain connectivity in dorsolateral prefrontal regions (Cole et al., 2011). Additional studies have focused on specific brain networks. For example, our previous work has examined connectivity within and between four replicable brain networks thought to be critical for cognitive function; (1) a "default mode" network (DMN; Damoiseaux et al., 2006; Raichle and Snyder, 2007) consisting of brain regions that reduce their activity during active cognitive demands; (2) a dorsal frontoparietal network (FP) activated by a range of cognitive control tasks (Dosenbach et al., 2006, 2007, 2008; Fair et al., 2007b); (3) a cingulo-opercular network (CO) thought to be involved in task set maintenance and error processing (Dosenbach et al., 2006, 2007, 2008; Fair et al., 2007b; Becerril et al., 2011); and (4) a cerebellar network (CER) that shows error related activity in many different types of tasks (Dosenbach et al., 2006, 2007, 2008; Fair et al., 2007b; Becerril et al., 2011). We found intact connectivity within each of these four networks among individuals with schizophrenia and their siblings, but reduced connectivity between all three control networks (FP, CO, and CER). Other studies have also found abnormal resting state connectivity in regions involved in the FP, CO, and CER networks (Zhou et al., 2007; Jafri et al., 2008; Welsh et al., 2008; Bassett et al., 2011; Woodward et al., 2011; Zalesky et al., 2011; Tu et al., 2012). Although we did not find functional connectivity changes with the DMN or between the DMN and other networks, other studies have found such alterations (Bluhm et al., 2007, 2009; Jafri et al., 2008; Whitfield-Gabrieli et al., 2009; Mannell et al., 2010; Rotarska-Jagiela et al., 2010; Salvador et al., 2010; Camchong et al., 2011; Chai et al., 2011).

Because these studies have measured connectivity at "rest," the typical interpretation has been that these changes in schizophrenia represent stable alterations in brain connectivity. However, one of the challenges of studying "rest" states is that connectivity changes could reflect differences in the cognitive states of the individuals, rather than stable structural or functional changes in brain connectivity. For example, if there were systematic differences in what individuals with schizophrenia were thinking about during rest (e.g., related to delusional or hallucinatory material, etc.; Sutton, 1973) or even during task states, this could lead to the appearance of altered functional connectivity. If such resting state changes in connectivity were due to such confounds, one might expect group differences in connectivity to be reduced (or at least altered) when participants were asked to engage in a specific task that imposed structure on the mental state of the individual. In contrast, if similar patterns of altered connectivity were found in resting state and during cognitive task performance in schizophrenia, it would provide support to the hypotheses that such connectivity changes reflect fundamental alterations in brain connectivity.

A number of studies have also examined functional connectivity during structured cognitive tasks in schizophrenia. A few of these studies have provided evidence for altered small-world characteristics during task states (Micheloyannis et al., 2006; Yu et al., 2011). However, most task-based functional connectivity studies have focused on specific regions or brain networks. These studies have provided evidence for alterations in functional connectivity across a range of tasks (Anticevic et al., 2011; Diaconescu et al., 2011; Fornito et al., 2011; Mukherjee et al., 2011), with working memory paradigms receiving the greatest focus. These studies have provided further evidence for connectivity changes in regions associated with the DMN, FP, CO, and CER networks, though the specific patterns of increased and decreased connectivity in schizophrenia have varied across studies (Meyer-Lindenberg et al., 2001, 2005; Schlosser et al., 2003a,b; Barch and Csernansky, 2007; Crossley et al., 2009; Henseler et al., 2010; Kang et al., 2011; White et al., 2011). Several of studies have either examined connectivity during a single task condition or integrated across conditions (Schlosser et al., 2003a,b; Barch and Csernansky, 2007; Crossley et al., 2009; Wolf et al., 2009), or found similar pattern of connectivity across task conditions (Meyer-Lindenberg et al., 2001). Such results could be consistent with the hypothesis that functional connectivity changes in schizophrenia reflect stable changes in brain connectivity. However, without a specific comparison to resting state conditions or between very different task conditions, it is difficult to know whether these are more state or trait related changes. Other studies have found evidence for different patterns of functional connectivity during different working memory task conditions (e.g., as a function of load, stimulus type, or task phase; Meyer-Lindenberg et al., 2005; Henseler et al., 2010; Kang et al., 2011; Rasetti et al., 2011). Such findings suggest that functional connectivity changes could reflect differences in task engagement or responsivity of brain networks to modulation, rather than stable changes that persist across all task states.

What is needed to help address these questions is a systematic examination of functional connectivity in schizophrenia across rest and across different task states to assess the degree to which altered functional connectivity reflects a trait like characteristic that is present regardless of the mental or behavioral state of the individual, or whether connectivity changes vary as a function of task demands. One previous fMRI study tried to examine this question in schizophrenia, comparing global brain connectivity across verb generation, an $N$-back working memory task, and rest (Salomon et al., 2011). These researchers found wide spread evidence for reduced functional connectivity, with the greatest differences during rest. However, interpretation of the results of this study are dramatically limited by the very small sample sizes, the fact that the same individuals did not participate in both the task and resting state experiments (meaning that state differences could reflect person differences), and by the failure to assess a number of potential methodological confounds (e.g., increased movement 
in patients). An EEG connectivity study also examined graph theoretic measures during rest and during a working memory task in schizophrenia. These researchers found reduced cluster coefficients among schizophrenia individuals during both rest and task in the alpha, beta and gamma bands, but only found increased path lengths during rest states (Micheloyannis et al., 2006).

The goal of the current study was to address questions related to the state dependence of functional connectivity changes in schizophrenia by comparing functional connectivity within and between four brain networks (DMN, FP, CO, and CER) during rest and during three different levels of working memory load between individuals with schizophrenia, their siblings, and healthy controls. We focused on working memory because of the consistent evidence for impairment in this cognitive domain in schizophrenia (Forbes et al., 2009), the fact that the largest number of task-based connectivity studies in schizophrenia have focused on working memory, and due to existing previous work in healthy individuals examining connectivity changes as a function of memory load during working memory. Specifically, Newton et al. (2011) recently demonstrated that brain regions within the FP and DMN networks showed increases in functional connectivity as working memory load increased, suggesting dynamic modulation of functional coupling among brain regions as task demands changes. We predicted that if functional connectivity changes in schizophrenia reflect stable changes in brain connectivity, we should see similar patterns of functional connectivity alterations across rest and across working memory loads. However, if at least some functional connectivity changes in schizophrenia reflect a failure to appropriately modulate brain networks as a function of changing task demands, then we may see increases in functional connectivity alterations in schizophrenia with increasing memory load or control demands.

\section{MATERIALS AND METHODS PARTICIPANTS}

The participants (Table 1) for this study were recruited through the Conte Center for the Neuroscience of Mental Disorders (CCNMD) at Washington University School of Medicine in St. Louis included: (1) individuals with DSM-IV Schizophrenia (SCZ; $N=19$ ); (2) the non-psychotic siblings of individuals with schizophrenia (SCZ-SIB; $N=28$ ); (3) healthy controls $(\mathrm{CON} ; N=10)$; and (4) the siblings of healthy controls (CON-SIB; $N=17$ ). Siblings were full siblings, based on self-report. These participants were a largely overlapping subset of participants reported on in our previous paper on resting state connectivity (Repovs et al., 2011) who had both resting state connectivity data and $N$-back working memory data. All participants gave written informed consent for participation and all participants had been included in our previous report on resting state functional connectivity changes in schizophrenia (Repovs et al., 2011). The average duration of illness for the individuals with schizophrenia was 4.79 ( $\mathrm{SD}=2.98$ ). Fifteen of the individuals with schizophrenia were taking atypical antipsychotics, and four were taking both typical and atypical antipsychotics.

All subjects were diagnosed on the basis of a consensus between a research psychiatrist who conducted a semi-structured interview and a trained research assistant who used the Structured Clinical
Interview for DSM-IV Axis I Disorders (First et al., 2001). Participants were excluded if they: (a) met DSM-IV criteria for substance dependence or severe/moderate abuse during the prior 6 months; (b) had a clinically unstable or severe medical disorder; (c) had a history of head injury with documented neurological sequelae or loss of consciousness; or (d) met DSM-IV criteria for mental retardation.

The individuals with schizophrenia were all outpatients, and were stabilized on antipsychotic medication for at least 2 weeks. Controls were required to have no lifetime history of Axis I psychotic or mood disorders and no first-degree relatives with a psychotic disorder. Potential SCZ-SIB subjects were excluded if they had a lifetime history of any DSM-IV Axis I psychotic disorder, but not other DSM-IV Axis I disorders. CON-SIB subjects were enrolled in an identical manner to SCZ-SIB subjects, and met the same general and specific inclusion and exclusion criteria, other than the requirement to have a sibling with schizophrenia. The siblings of healthy controls had the following diagnoses: (1) previous substance abuse $(N=4,24 \%)$; (2) previous substance dependence $(N=1,6 \%)$; (3) major depression $(N=3,18 \%)$; and (4) social phobia $(N=1,6 \%)$. The siblings of schizophrenia patients had the following diagnoses: (1) previous substance abuse $(N=6,21 \%)$; (2) previous substance dependence $(N=2,7 \%)$; (3) bipolar II disorder $(N=1,7 \%)$; (4) major depression $(N=6$, $21 \%)$; (5) social phobia ( $N=1,7 \%)$; and (6) PTSD $(N=1,7 \%)$.

\section{CLINICAL AND COGNITIVE ASSESSMENTS}

Psychopathology and cognitive function outside of the MR scanner were assessed as previously described (Delawalla et al., 2006; Harms et al., 2007) and as described in the Supplemental Materials. Scores for each symptom domain and each cognitive domain are shown in Table 1.

\section{fMRI SCANNING}

All scanning occurred on a 3T Tim TRIO Scanner at Washington University Medical School. Functional images (BOLD) were acquired using an asymmetric spin-echo, echo-planar sequence $\left[\mathrm{T}_{2}^{*}\right.$; repetition time $(\mathrm{TR})=2500 \mathrm{~ms}$, echo time $(\mathrm{TE})=27 \mathrm{~ms}$, field of view $(\mathrm{FOV})=256 \mathrm{~mm}$, flip $=90^{\circ}$, voxel size $=4 \mathrm{~mm} \times 4 \mathrm{~mm} \times 4 \mathrm{~mm}$ ]. Resting state data were acquired from each participant for two BOLD runs in which participants rested quietly with their eyes closed. Each run contained 164 images, for a total of 328 images and $13.7 \mathrm{~min}$ of resting state activity. Working memory task data were acquired from each participant in three BOLD runs, each consisting of two blocks of 0-back, 1-back, or 2-back working memory task. Each run consisted of 137 images (105 of them acquired during task performance), for a total of 411 images (315 acquired during task performance) and $17.1 \mathrm{~min}$ of scanning (13.1 min of task performance). In addition, a T1 structural image was acquired using a sagittal MP-RAGE $3 \mathrm{D}$ sequence $(\mathrm{TR}=2400 \mathrm{~ms}$, TE $=3.16 \mathrm{~ms}$, flip $=8^{\circ}$; voxel size $\left.=1 \mathrm{~mm} \times 1 \mathrm{~mm} \times 1 \mathrm{~mm}\right)$.

\section{N-BACK WORKING MEMORY TASK}

All subjects performed one run of each of three levels of an " $N$ back" working memory task in which their task was to respond for each letter shown whether it was the same as a pre-specified 
Table 1 | Demographic, clinical, and performance characteristics of study participants.

\begin{tabular}{|c|c|c|c|c|c|c|c|c|}
\hline \multirow[t]{3}{*}{ Measure } & \multicolumn{8}{|c|}{ Group } \\
\hline & \multicolumn{2}{|c|}{$\begin{array}{l}\text { Healthy controls } \\
\text { (CON) }\end{array}$} & \multicolumn{2}{|c|}{$\begin{array}{l}\text { Siblings of controls } \\
\text { (CON-SIB) }\end{array}$} & \multicolumn{2}{|c|}{$\begin{array}{l}\text { Individuals with } \\
\text { schizophrenia (SCZ) }\end{array}$} & \multicolumn{2}{|c|}{$\begin{array}{l}\text { Siblings of schizophrenia } \\
\text { (SCZ-SIB) }\end{array}$} \\
\hline & Mean & SD & Mean & SD & Mean & SD & Mean & SD \\
\hline Age & 24.3 & 2.7 & 22.6 & 2.8 & 24.4 & 3.4 & 24.2 & 3.6 \\
\hline Parental education & 15.3 & 1.7 & 14.4 & 1.5 & 14.2 & 2.1 & 15 & 2.3 \\
\hline Negative symptoms* & -0.42 & 0.20 & -0.33 & 0.42 & $1.13^{a}$ & 0.75 & -0.13 & 0.53 \\
\hline Positive symptoms* & -0.51 & 0.10 & -0.32 & 0.41 & $1.08^{\mathrm{a}}$ & 1.10 & -0.27 & 0.30 \\
\hline Disorganization symptoms* & -0.33 & 0.28 & -0.26 & 0.25 & $0.73^{a}$ & 0.98 & -0.15 & 0.35 \\
\hline \multicolumn{9}{|l|}{ N-BACK PERFORMANCE } \\
\hline $1 \mathrm{Q}^{\wedge}$ & 0.21 & 0.54 & -0.42 & 0.76 & $-0.91^{a}$ & 0.67 & -0.34 & 0.76 \\
\hline Working memory^ & 0.82 & 0.66 & 0.53 & 0.56 & $-0.52^{a}$ & 0.63 & $0.23^{c}$ & 0.72 \\
\hline Episodic memory^ & 0.74 & 0.84 & 0.44 & 0.53 & $-0.89^{a}$ & 0.44 & $-0.03^{b}$ & 0.57 \\
\hline Executive function^ ${ }^{\wedge}$ & -0.76 & 0.38 & 0.55 & 0.39 & $-0.38^{a}$ & 0.85 & $-0.14^{b}$ & 0.47 \\
\hline
\end{tabular}

The four groups did not differ significantly in age $[F(3,70)=1.2, p>0.10]$, parental education $[F(3,70)=0.96, p>0.50]$, gender $\left(X^{2}=2.5, p>0.4\right)$, or race $\left(X^{2}=7.2\right.$, $p>0.3)$. The groups did differ in personal education $[F(3,70)=4.06, p<0.05]$, with the SCZ having fewer years of education than CON. The NS for SCZ and SCZ-SIB and for CON and CON-SIB are not identical given that some participants were excluded for failure to complete the entire protocol or excessive movement. Of the 101 participants aged 18 or older from whom we collected resting state data, there were 12 who had to be excluded for poor quality imaging data (4 SCZ, 4 SCZ-SIB, 1 CON, and 3 CON-SIB) and 15 who were excluded because they did not have N-back data (6 SCZ, 3 SCZ-SIB, 5 CON, and 1 CON-SIB).

*Symptom scores are reported in Z scores relative to the mean of the entire sample. See Section "Materials and Methods" for details. One-way ANOVAs indicated significant group differences for positive $[F(3,70)=25.14, p<0.001]$, negative $[F(3,70)=3-0.88, p<0.001]$, and disorganization $[F(3,70)=13.57, p<0.001]$ symptoms. ${ }^{a}$ Post hoc contrasts using Tukey's HSD indicated that the SCZ had higher scores on all three symptom domains, with no significant differences among the remaining groups.

${ }^{\wedge}$ Cognitive scores are reported in Z scores relative to the mean of the entire sample. See Section "Materials and Methods" for details. One-way ANOVAs indicated significant group differences for $1 Q[F(3,70)=5.69, p<0.001]$, working memory $[F(3,70)=12.06, p<0.001]$, episodic memory $[F(3,70)=23.7, p<0.001]$, and executive function $[F(3,70)=11.96, p<0.001]$. ${ }^{a}$ Post hoc contrasts using Tukey's HSD indicated that the SCZ participants had worse performance in all four cognitive domains than CON and CON-SIB. ${ }^{b} S C Z-S I B$ performed worse than CON on executive function and episodic memory, and showed a ${ }^{\circ}$ trend for reduced performance on working memory $(p<0.10)$.

letter (e.g., "X"; 0-back), the same as the immediately preceding letter (1-back), or the same as the letter shown two trials previously (2-back). Each of the memory loads was performed for two task blocks within the same run and the order of runs was counterbalanced across participants. The task followed a mixed state-item design. Each block started with a cue shown for $2.5 \mathrm{~s}$ indicating the $N$-back condition, followed by letters presented one at a time for $2.5 \mathrm{~s}$ each. The delay between items was variable with the following proportion of delays 1 TR: 5\%, 2 TR: 31\%, and 3 TR: 64\%. Each task block contained 21 trials and lasted for a total of $105 \mathrm{~s}$. Each run started with $25 \mathrm{~s}$ of fixation, and each task block was followed by a 45 s fixation block.

\section{fCMRI DATA PREPROCESSING}

Basic imaging data preprocessing included: (1) Compensation for slice-dependent time shifts; (2) Removal of first five images from each run during which BOLD signal was allowed to reach steady state; (3) Elimination of odd/even slice intensity differences due to interpolated acquisition; (4) Realignment of data within and across runs to compensate for rigid body motion (Ojemann et al., 1997); (5) Intensity normalization to a whole brain mode value of 1000; (6) Registration of the 3D structural volume (T1) to the atlas representative template in the Talairach coordinate system (Talairach and Tournoux, 1988) using a 12-parameter affine transform; and (7) Co-registration of the 3D fMRI volume to the structural image and transformation to atlas space using a single affine 12-parameter transform that included a re-sampling to a 3-mm cubic representation.

To improve signal-to-noise, remove baseline, possible sources of spurious correlations, and task structure, all images were further preprocessed in steps that included: (1) spatial smoothing using a gaussian kernel with three voxels FWHR, (2) high-pass filtering with $0.009 \mathrm{~Hz}$ cutoff frequency ${ }^{1}$, (3) removal of nuisance

${ }^{1}$ In prior work Repovs et al. (2011), we compared using just a high-pass filter and both a high and low-pass filter, finding identical results with both approaches. Thus, 
signal that included six rigid body motion correction parameters, ventricle, white matter, and whole brain signals, as well as their first derivatives. In task data the additional regressors included sustained task activity, modeled using assumed-response Boynton HRF function (Boynton et al., 1996), and transient response activity, modeled as unassumed response spanning nine frames. Task response was modeled separately for each of the task levels. All connectivity analyses were conducted on residual timeseries after removal of listed regressors.

A frequent confound in imaging studies with clinical populations is that the clinical group moves more, which can lead to lower signal-to-noise ratio (SNR) in the acquired resting state data, and perhaps also apparent reductions in connectivity. Thus, we took two approaches to addressing movement-related confounds. First, we directly compared the four groups on average SNR during the resting state runs and during the working memory runs. SNR was computed as the mean value for each slice across each run, divided by the standard deviation across the frames in the run. We then averaged the SNR values across slices and runs within either resting state or working memory. We used a repeated measures ANOVA with condition (rest versus task) as a within subject factor, and genetic liability as a between subject factor. There was a significant main effect of condition $[F(1,70)=20.4, p<0.001]$, with lower SNR in rest versus task. This finding is consistent with the fact that movement tends to increase the longer individuals are in the MR scanner, and participants completed the resting state scans after the working memory scans. There was no significant main effect of genetic liability $[F(3,70)=1.3, p=0.27]$, and no significant interaction between condition and genetic liability $[F(3$, $70)=2.2, p=0.09]$. Second, as a last preprocessing step, frames with excessive movement and movement-related intensity changes were identified and excluded from further analysis. Bad frames were identified following a modified procedure suggested by Power et al. (2011) as those that met at least one of the two criteria. First, frames in which sum of the displacement across all six rigid body movement correction parameters exceeded $0.5 \mathrm{~mm}$ were identified. Second, root mean square (RMS) of differences in intensity between the current and preceding frame was computed across all voxels and divided by mean intensity. Frames in which normalized RMS was more than 1.6 the median across the run were identified. The identified frames, one preceding and two following frames were then marked for exclusion in computation of functional connectivity. A repeated measures ANOVA for the percentage of eliminated frames, with run type (rest, working memory) as a within subject factor and group (SCZ, SCZ-SIB, CON, CON$\mathrm{SIB}$ ) as a between subject factor, indicated a main effect of group $[\mathrm{F}(3,70)=3.98, p<0.05]$, but no main effect of run type $[\mathrm{F}(1$, $70)=0.02, p>0.5]$ and no interaction between group and run type $[\mathrm{F}(3,70)=0.43, p>0.5]$. Post hoc tests using Tukey's HSD indicated that SCZ $(M=9.4 \%, \mathrm{SD}=7.7 \%)$ had more eliminated trials than CON-SIB $(M=1.6 \%, \mathrm{SD}=2.2 \%)$, but no further significant differences from either SCZ-SIB $(M=6.2 \%, \mathrm{SD}=9.11 \%)$ or $\operatorname{CON}(M=3.6 \%, \mathrm{SD}=3.0 \%)$.

we used only the high-pass filter approach here to be consistent with our prior resting study connectivity study on these individuals.
For resting state data, the two BOLD timeseries (excluding the first five frames) were concatenated to form a single timeseries. For task-based data, only task-related parts of the BOLD timeseries were used. Due to the delay in HRF, the first three frames after task onset were excluded, and one frame after the end of task block were included in the timeseries. The initial BOLD preprocessing was accomplished using inhouse software, fcMRI preprocessing and analyses described below were performed using custom Matlab (The Mathworks, Natick, Massachusetts) code.

\section{NETWORK REGION DEFINITION}

We examined regions included in the DMN as defined by Fox et al. (2005), and regions included in the FP, CO, and CER networks as defined by Dosenbach et al. (2007). To control for individual anatomical variability, ROI were defined for each individual in two steps. First, we created spherical ROIs in standard Talairach space centered on the reported coordinates for each region (Figure 1; Table S1 in Supplementary Material) and $15 \mathrm{~mm}$ in diameter. Second, we masked the resulting group ROIs with the individual FreeSurfer (http://surfer.nmr.mgh.harvard.edu, version 4.1.0) segmentation of a high-resolution structural image that was previously registered to standard Talairach space, excluding any voxels within the group defined ROIs that did not represent the relevant gray matter (cerebral cortex, cerebellar cortex, hippocampus, thalamus) in the specific individual, as defined by FreeSurfer (Fischl et al., 2002). Given that we used a priori ROIs, we conducted a number of analyses in the control subjects using these ROIs to validate the expected pattern of connectivity within and between these four networks. The results of these analyses are presented in the supplement.

\section{DATA ANALYSIS}

We extracted the time series for each of the ROIs described above and computed the ROI-ROI correlation matrix for each participant, separately for resting state (R), and 0-back (0B), 1-back (1B) and 2-back (2B) task-based data. We then converted correlations to Fisher $z$-values using Fisher $r$-to- $z$ transform and computed the average connectivity (mean Fisher $z$-value) across all ROIROI connections within each of the four networks, and computed the average connectivity across all ROI-ROI connections between each network. We denoted within-network averages as wDMN, wFP, wCO, and wCER, and between network connectivity averages as bDMN-FP, bDMN-CO, bDMN-CER, bFP-CO, bFP-CER, and bCO-CER. We estimated group-level statistical significance by using the resulting Fisher- $z$ values as the dependent measure in the second-level analysis.

To compare the groups and assess the effect of task on connectivity within and between networks we analyzed the results in two phases. First, we focused on comparison between rest and task $(0 \mathrm{~B})$, and second, on the effect of working memory load $(0 \mathrm{~B}, 1 \mathrm{~B}, 2 \mathrm{~B})$ in both cases using separate mixed design ANOVAs for exploring within and between network connectivity. In the ANOVA presented below, we include a between subject factor that we call genetic liability, to indicate that the participants were either individuals with schizophrenia or their siblings (SCZ) or healthy controls or their siblings (CON). Thus, these ANOVAs 


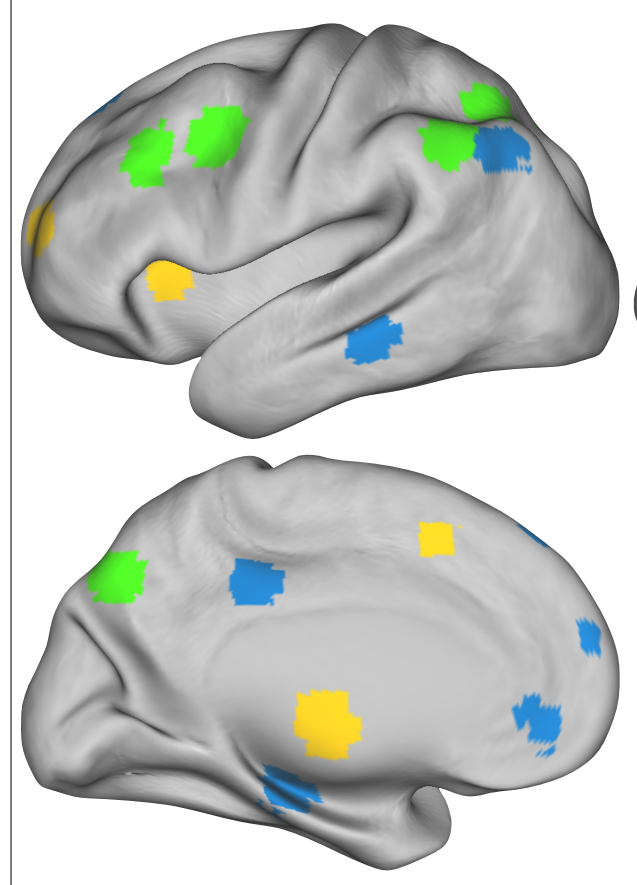

FIGURE 1 | Figure illustrating the location of regions within each of the four networks. Regions of the Frontal-Parietal network (FP) are marked in
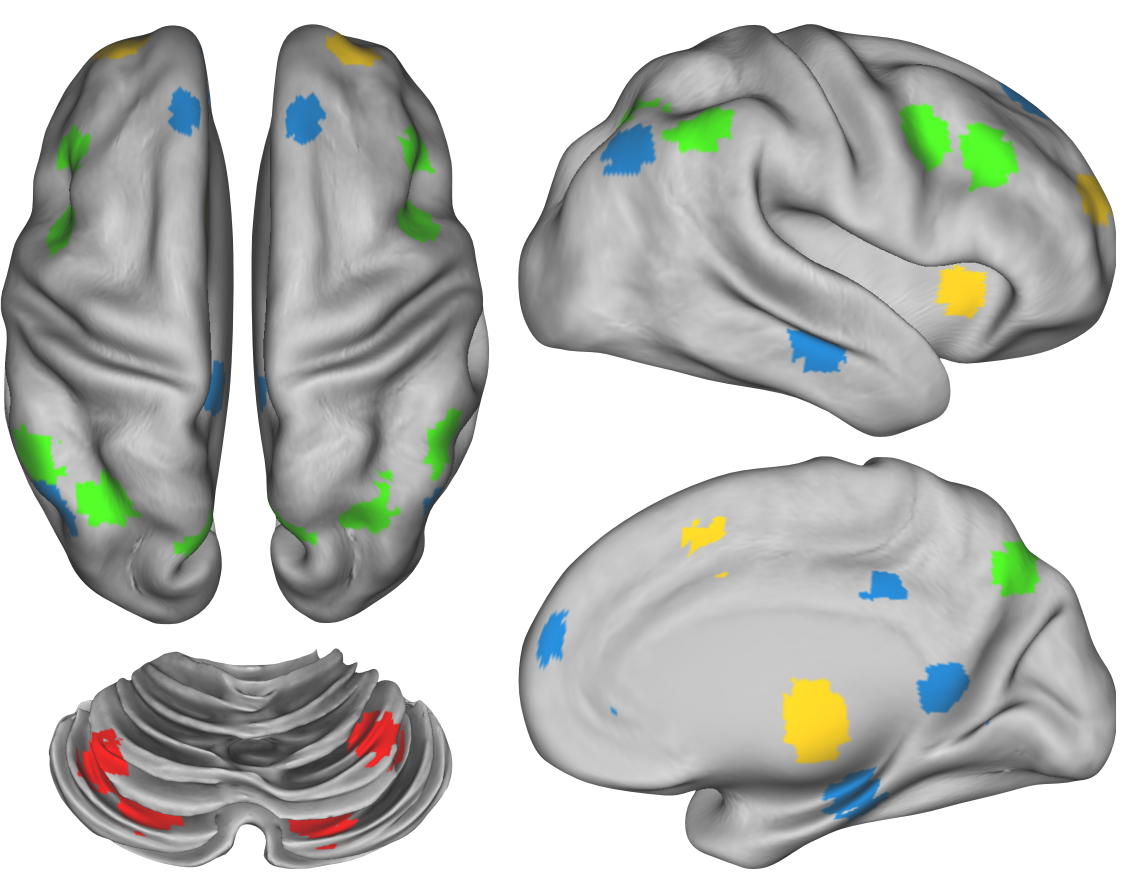

green, the Cingulo-Opercular network (CO) in yellow, the Default Mode Network (DMN) in blue, and the Cerebellar network (CER) in red. included genetic liability (SCZ versus $\mathrm{CON}$ ) and family member type [index (i.e., SCZ or CON) versus sibling (i.e., SCZ-SIB or CON-SIB)] as between subject factors. Significant effects were further explored with planned comparison, using False Discovery Rate to control for multiple comparisons, to isolate the source of significant ANOVA effects. For the sake of brevity, we do not report main effects or interactions that include family member type, but do not also include genetic liability. Statistical analysis was conducted using R (Team, 2011) and visualized using ggplot2 library (Wickham, 2009).

\section{RESULTS}

\section{BEHAVIORAL DATA}

The accuracy data for the $N$-back task was analyzed using a repeated measures ANOVA with load $(0 \mathrm{~B}, 1 \mathrm{~B}, 2 \mathrm{~B})$ as a within subject factor, and genetic liability ( $\mathrm{SCZ}$ or $\mathrm{CON}$ ) and family member type (index or sibling) as between subject factors. As shown in Table 1, this ANOVA revealed a main effect of load $[F(2,140)=4.76, p<0.01]$, a main effect of genetic liability $[F(1$, $70)=7.48, p<0.01]$, a main effect of family member type $[F(1$, $70)=7.05, p=0.01]$, and a trend level load by genetic liability interaction $[F(2,140)=2.4, p=0.09]$. Accuracy decreased as memory load went up, and the SCZ and SCZ-SIB performed worse than the CON and CON-SIB. The main effect of sibling group indicated that the index siblings performed overall worse. The reaction time data (median correct) also showed a significant main effect of load $[F(2,140)=92.0, p<0.001]$, but no other significant main effects or interactions. Reaction times increased as a function of memory load across all groups.

\section{TASK VERSUS REST AND WITHIN-NETWORK CONNECTIVITY}

The within-network ANOVA for task versus rest included genetic liability (SCZ or CON) and family member type (index or sibling) as between subject factors, and task [0-back (OB) versus rest (R)] and network (wDMN, wFP, wCO, and wCER) as within subject factors. This ANOVA revealed a significant effect of task $[\mathrm{F}(1,70)=7.82, p=0.006]$, a significant effect of network $[\mathrm{F}(3$, $310)=52.2, p<0.001]$, and a significant task $\times$ network interaction $[\mathrm{F}(3,210)=20.6, p<0.001]$. There were no significant effects of genetic liability, though there was a trend for a threeway interaction between task, network, and genetic liability $[\mathrm{F}(3$, $210)=2.29, p=0.08$ ]. To examine the source of task $\times$ network interaction we ran separate ANOVAs for each of the four networks employing FDR correction for multiple comparison across the tests. The results revealed a significant effect of task for all four networks. However the pattern of this effect differed across networks (see Figure 2 for graphs collapsing across SCZ and SCZ-SIB and CON and CON-SIB; See Figure S4 in Supplementary Material for data plotted for each group individually). There was stronger connectivity within the $\operatorname{DMN}[\mathrm{F}(1,70)=10.4$, $p=0.002]$ and the FP $[\mathrm{F}(1,70)=4.60, p=0.035]$ networks for $\mathrm{OB}$ as compared to rest. In contrast, there was weaker connectivity within the $\mathrm{CO}[\mathrm{F}(1,70)=8.54, p=0.005]$ and the CER $[\mathrm{F}(1,70)=31.7, p=0.001]$ networks for $0 \mathrm{~B}$ compared to rest. The three-way interaction between network, task, and genetic liability was at trend level. This reflected the fact that the followup contrasts indicated a task $\times$ genetic liability interaction in $\mathrm{wDMN}[\mathrm{F}(1,70)=4.15, p=0.045]$ connectivity. Although this effect would not survive FDR correction for multiple comparisons, 


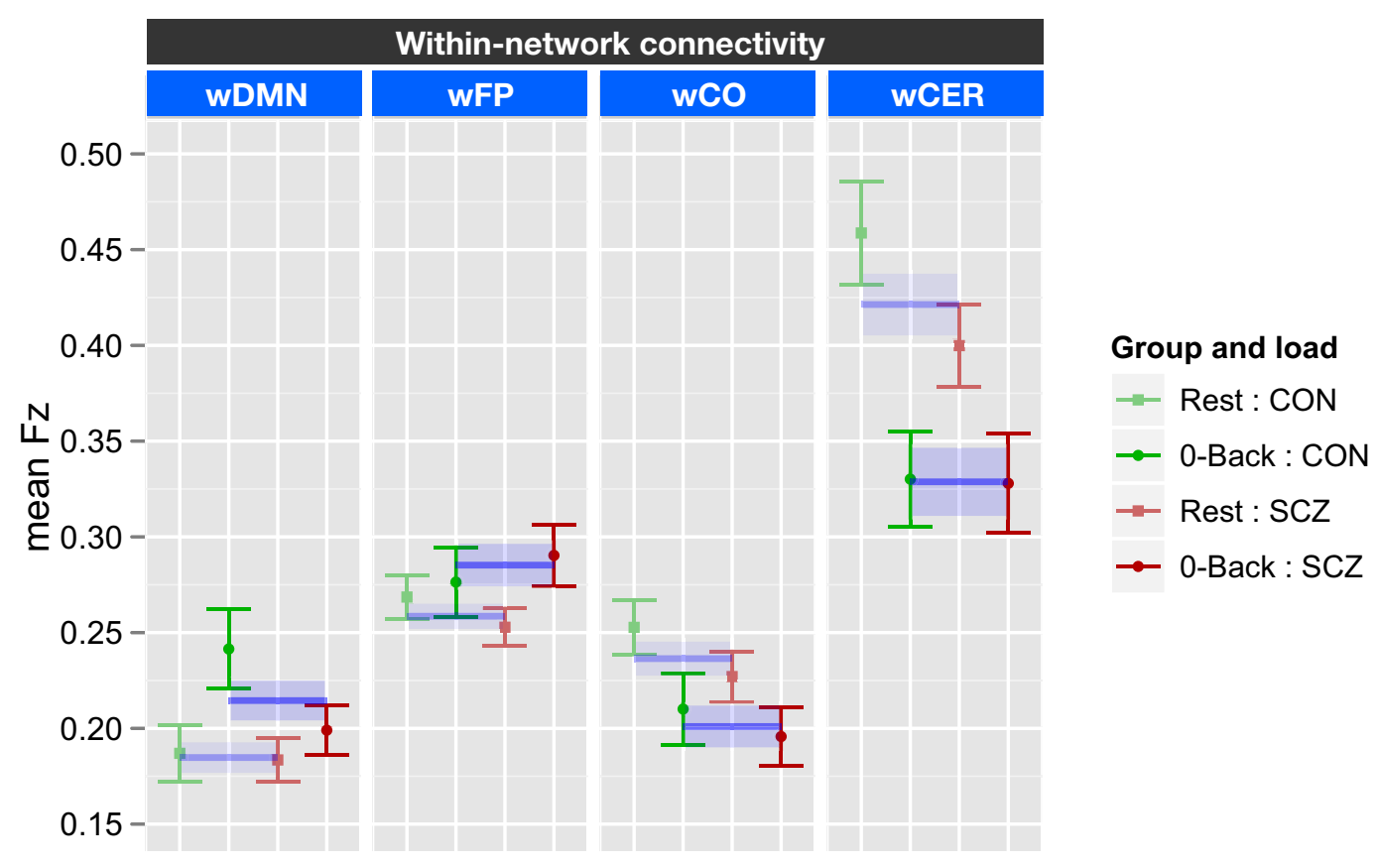

FIGURE 2 | Graph illustrating 0-back task versus rest within-network connectivity collapsed across siblings. $S C Z$, individuals with schizophrenia and siblings of individuals with schizophrenia; CON, healthy controls and siblings of healthy controls; DMN, Default Mode Network; FP, Frontal-Parietal Network; CO, Cingulo-Opercular Network; CER, Cerebellar Network; w, within. Segments marked in blue indicate networks that showed significant main effects of task (0-back versus rest). The main effect of task is further illustrated by blue lines and shading showing data collapsed across all groups (mean and standard error). See Figure S4 in Supplementary Material for data plotted for each of the four groups separately. it suggested that SCZ and SCZ-SIB did not show a significant difference between $0 \mathrm{~B}$ and rest in $\mathrm{WDMN}$ connectivity. No other networks demonstrated a significant task by group interaction (all ps $>0.10)$.

\section{TASK VERSUS REST AND BETWEEN NETWORK CONNECTIVITY}

The between network ANOVA for task versus rest also included genetic liability and family member type as between subject factors, and task (0B versus rest) and network (bDMN-FP, bDMNCO, bDMN-CER, bFP-CO, bFP-CER, and bCO-CER) as within subject factors. This ANOVA revealed significant main effects of genetic liability $[\mathrm{F}(1,70)=14.1, p<0.001]$, network $[\mathrm{F}(5$, $350)=46.1, p<0.001]$, and task $[\mathrm{F}(1,70)=17.1, p<0.001]$ as well as significant task $\times$ network $[\mathrm{F}(5,350)=5.6, p<0.001]$ and network $\times$ genetic liability $[\mathrm{F}(5,350)=4.07, p=0.001]$ interactions. To follow-up on the significant effects, we ran ANOVAs for each of the six between network connectivities employing FDR correction for multiple comparisons (see Figure 3). The significant network by task interaction reflected the fact that there was a significant effect of task for bDMN-FP $[\mathrm{F}(1,70)=26.6$, $p<0.0001]$, bDMN-CO $[\mathrm{F}(1,70)=12.9, p<0.001]$, and bDMN$\operatorname{CER}[\mathrm{F}(1,70)=14.1, p<0.001]$, but not for the other between network connections. The connectivity between the DMN and the other three networks was reduced in $0 \mathrm{~B}$ versus rest (Figure 3). The significant network $\times$ genetic liability interaction reflected the fact that there was a significant effect of genetic liability for bFP$\operatorname{CER}[\mathrm{F}(1,70)=13.9, p<0.001]$ and bCO-CER $[\mathrm{F}(1,70)=15.2$, $p<0.001]$ connectivity, but not for the other between network connections. For both bFP-CER and bCO-CER, connectivity was overall lower in patients and their siblings compared to controls and their siblings (see Figure 3; Figure S5 in Supplementary Material).

\section{WORKING MEMORY LOAD AND WITHIN-NETWORK CONNECTIVITY}

The within-network ANOVA for working memory load again included genetic liability and family member type as between subject factors, and working memory load $(0 \mathrm{~B}, 1 \mathrm{~B}, 2 \mathrm{~B})$ and network (wDMN, wFP, wCO, and wCER) as within subject factors. This ANOVA revealed a significant main effect of network $[\mathrm{F}(3,210)=45.6, p<0.001]$ and a significant load $\times$ network interaction $[\mathrm{F}(6,420)=6.39, p<0.001]$. The main effect of genetic liability was not significant $[\mathrm{F}(1,70)=0.07, p>0.9]$ and there were no significant interactions with genetic liability (all $p s>0.15)$. To determine the source of the load $\times$ network interaction, we computed separate ANOVAs for each of the four networks and employed FDR for multiple comparison correction. These ANOVAs revealed significant, but opposite effects, of load on connectivity within the $\operatorname{DMN}[\mathrm{F}(2,140)=13.4, p<0.0001]$ and $\mathrm{FP}$ $[\mathrm{F}(2,140)=13.0, p<0.0001]$ networks. Connectivity decreased with increasing load within DMN, but increased with load within FP network (see Figure 4; Figure S6 in Supplementary Material). We should again note that while the network $\times$ load $\times$ genetic liability interaction was not significant, the follow-up contrasts did indicate a genetic liability $\times$ load interaction in wDMN connectivity $[\mathrm{F}(2,140)=3.67, p=0.028]$. This effect would not survive FDR correction for multiple comparisons, but did reflect the fact 


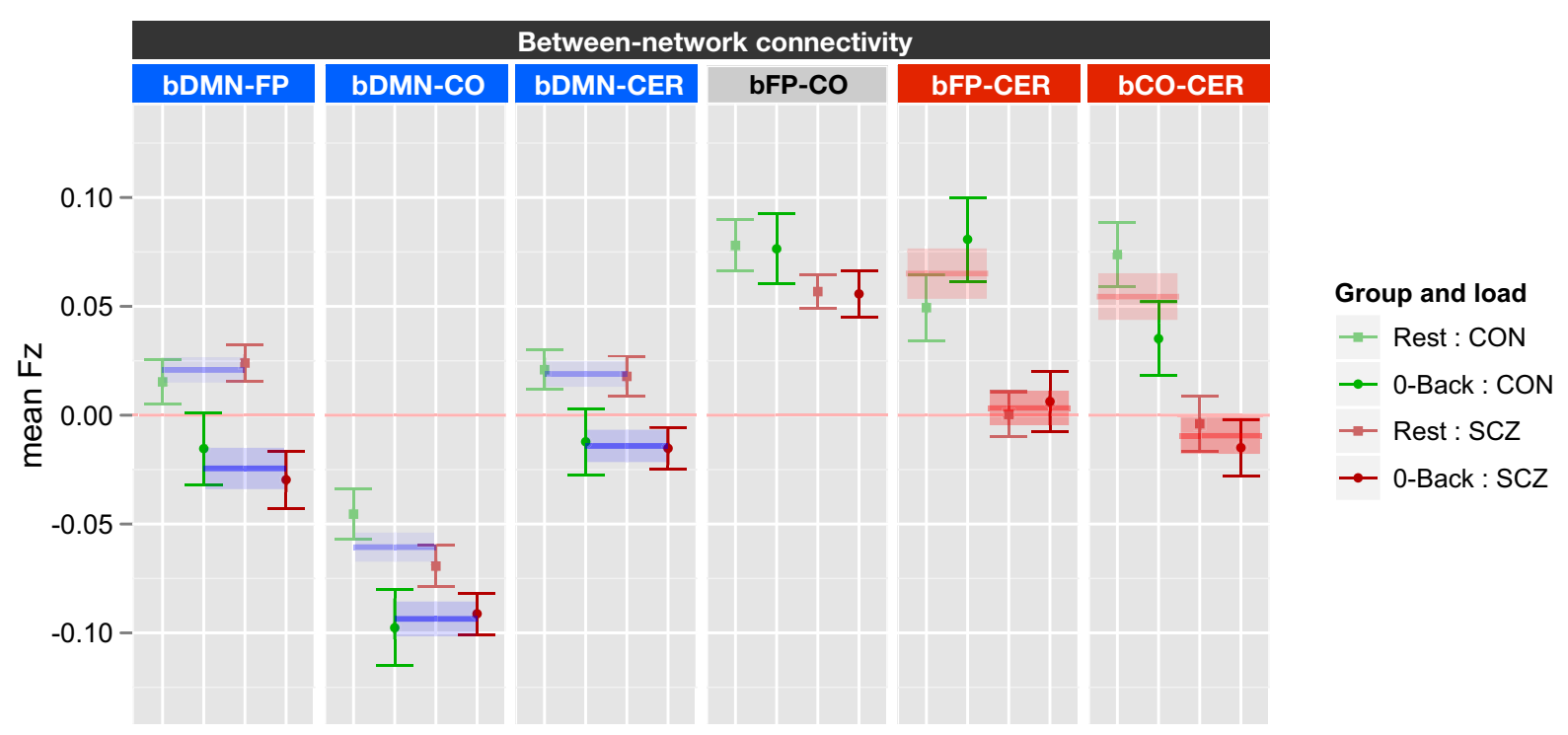

FIGURE 3 | Graph illustrating 0-back versus rest between network connectivity collapsed across siblings. SCZ, individuals with schizophrenia and siblings of individuals with schizophrenia; CON, healthy controls and siblings of healthy controls; DMN, Default Mode Network; FP, Frontal-Parietal Network; CO, Cingulo-Opercular Network; CER, Cerebellar Network; b, between. Segments marked in blue indicate networks which showed significant main effects of task (0-back versus rest). The main effect of task is further illustrated by blue lines and shading showing data collapsed across all groups (mean and standard error). Segments marked in red indicate networks that showed a significant main effect of genetic liability (SCZ versus CON).

The main effect of genetic liability is further illustrated by red lines and shading showing data collapsed across task conditions (mean and standard error for each group across task and rest). See Figure S5 in Supplementary Material for data plotted for each of the four groups separately.

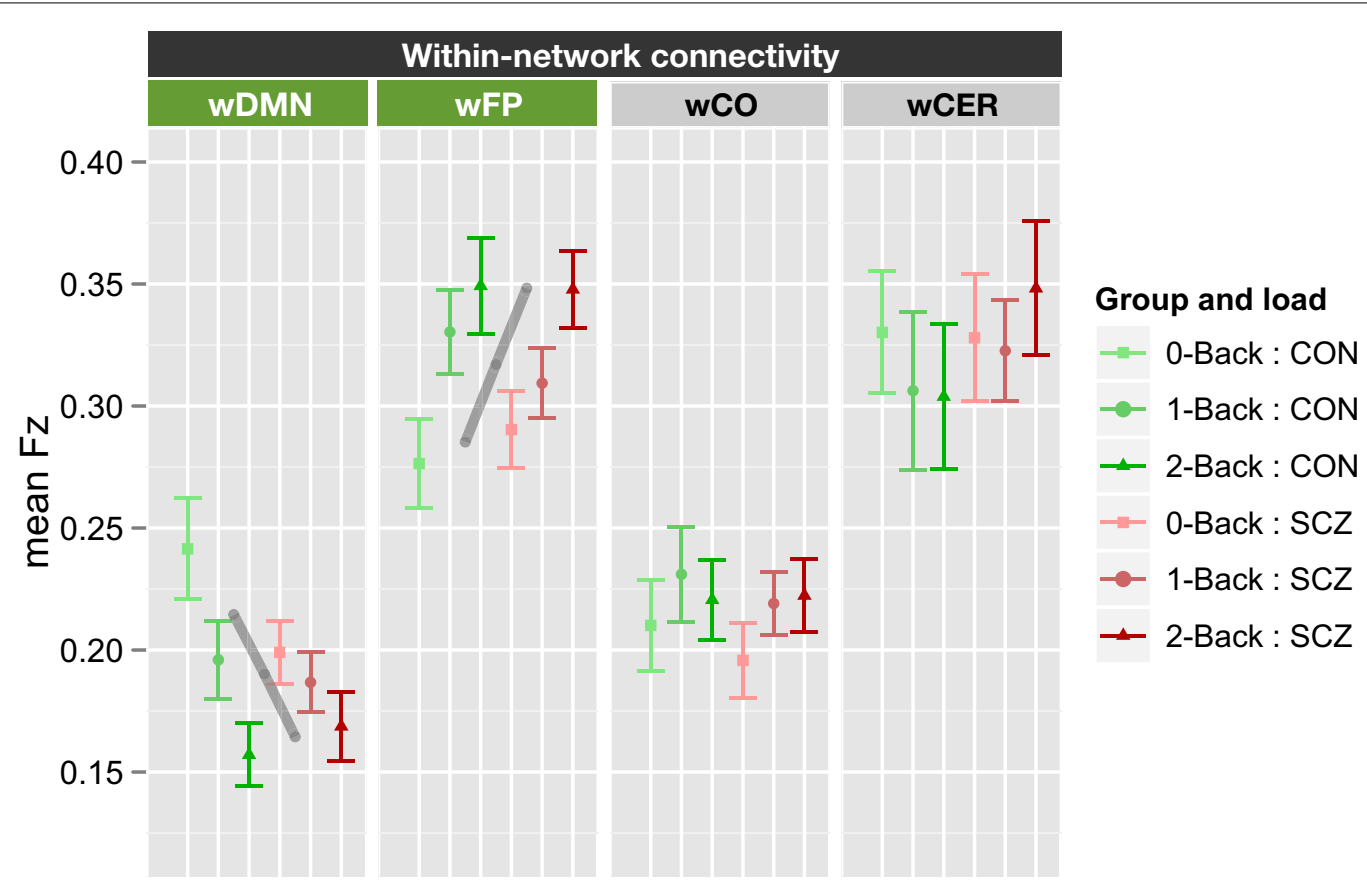

FIGURE 4 | Graph illustrating within-network connectivity as a function of working memory load collapsed across siblings. SCZ, individuals with schizophrenia and siblings of individuals with schizophrenia; CON, healthy controls and siblings of healthy controls; DMN, Default Mode Network; FP, Frontal-Parietal Network; CO, Cingulo-Opercular Network; CER, Cerebellar
Network; w, within. Segments marked in green indicate networks that showed significant main effects of working memory load $(0 \mathrm{~B}, 1 \mathrm{~B}, 2 \mathrm{~B})$ and the gray lines further illustrate the significant main effect of load across groups (mean across groups). See Figure S6 in Supplementary Material for data plotted for each of the four groups separately. 
that the decrease in wDMN connectivity as a function of memory load was less in SCZ and SCZ-SIB than in CON and CON-SIB.

\section{WORKING MEMORY LOAD AND BETWEEN NETWORK CONNECTIVITY}

Finally, the effects of working memory load and genetic liability on between network connectivity was tested using the same ANOVA design as for within-network connectivity. The results revealed significant main effects of genetic liability $[\mathrm{F}(1,70)=16.1$, $p<0.001]$, network $[\mathrm{F}(5,350)=64.1, p<0.001]$, and working memory load $[\mathrm{F}(2,140)=12.2, p<0.001]$. In addition, the genetic liability $\times$ network $[\mathrm{F}(5,350)=2.48, p=0.031]$ and working memory load $\times$ network $[\mathrm{F}(10,700)=6.17, p<0.001]$ interactions were also significant. To examine the source of the working memory load $\times$ network interaction, we conducted separate ANOVAs for each of the six between network connections, using FDR to control for multiple comparisons. These analyses revealed significant effects of working memory load on bDMN-CO $[\mathrm{F}(2$, $140)=7.9, p<0.001]$, bFP-CO $[\mathrm{F}(2,140)=21.4, p<0.0001]$, and bFP-CER $[\mathrm{F}(2,140)=7.93, p<0.001]$ connectivity, but not for the other between network connections. The significant genetic liability $\times$ network interaction reflected the fact that we also observed significant main effects of genetic liability for bFP-CER $[\mathrm{F}(1$, $70)=14.1, p<0.001]$ and bCO-CER $[\mathrm{F}(1,70)=8.78, p=0.004]$ connectivity, but not for the other between network connections. For both bFP-CER and bCO-CER, connectivity was overall lower in SCZ and their siblings compared to CON and their siblings (see Figure 5; Figure S7 in Supplementary Material). Importantly, however, these effects did not further interact with load. Thus, even though connectivity was overall lower for bFP-CER (which showed a main effect of working memory load) in SCZ and SCZ-SIB, SCZ, and SCZ-SIB still showed an increase in bFP-CER connectivity as load increased.

\section{RELATIONSHIP TO CLINICAL AND COGNITIVE VARIABLES}

In our previous work on resting state connectivity in this sample, we had found that bFP-CER connectivity (which was lower in SCZ and SCZ-SIB) predicted neuropsychological performance on IQ, working memory, episodic memory, and executive function assessed outside of the scanner. In addition, bFP-CER predicted disorganization symptoms. Thus, we wished to examine whether connectivity during task also predicted cognitive function, either on the working memory task performed in the scanner, or on the neuropsychological measures assessed outside of the scanner. We focused on the connectivity measures that differed between groups: bFP-CER, bFP-CO, and wDMN. For task connectivity, we examined the average connectivity across working memory loads, since none of the significant group differences interacted with load. We computed partial correlations between the connectivity measures and the cognitive and clinical measures, controlling for group status. Neither the resting state nor task connectivity measures for DMN displayed any significant correlations. However, as shown in Table 2, resting state bFP-CER connectivity again predicted better cognitive performance (working memory, episodic memory, and executive function) and fewer disorganization symptoms, though these are not new results given that this sample closely resembled the one in our prior study. As can be seen in Figure S9 in Supplementary Material, the relationship between bFP-CER and cognitive performance are consistent across groups. In contrast, as

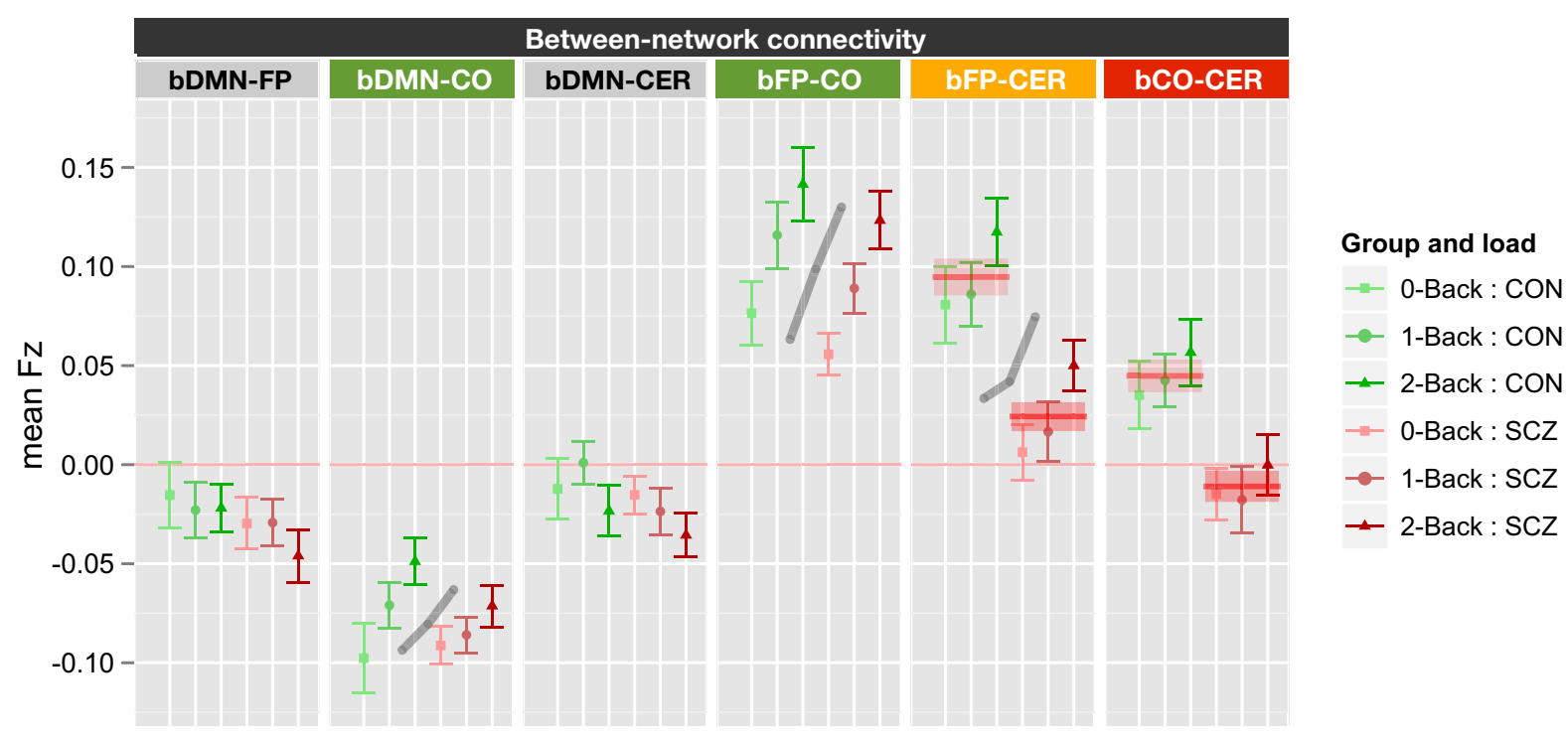

FIGURE 5 | Graph illustrating between network connectivity as a function of working memory load collapsed across siblings. SCZ, individuals with schizophrenia and siblings of individuals with schizophrenia; CON, healthy controls and siblings of healthy controls; DMN, Default Mode Network; FP, Frontal-Parietal Network; CO, Cingulo-Opercular Network; CER, Cerebellar Network; b, between. Segments marked in green indicate networks that showed significant main effects of working memory load (OB, $1 B, 2 B$ ) and the gray lines further illustrate the significant main effect of load across groups (mean across groups). Segment marked in orange showed both a significant main effect of load and a significant main effect of genetic liability (SCZ versus CON). The segment marked red indicates the network that showed only a significant main effect of genetic liability (SCZ versus CON). The red lines further illustrate the statistically significant effects of genetic liability (mean and standard error for each group across memory loads). See Figure S7 in Supplementary Material for data plotted for each of the four groups separately. 
Table 2 | Correlations between connectivity measures and cognitive and clinical variables.

\begin{tabular}{|c|c|c|c|c|}
\hline & \multicolumn{4}{|c|}{ Connectivity type } \\
\hline & Resting & Resting & Task & Task \\
\hline & FP to CER connectivity & CO to CER connectivity & FP to CER connectivity & CO to CER connectivity \\
\hline \multicolumn{5}{|c|}{$N$-BACK PERFORMANCE $(r)$} \\
\hline 0-back & 0.20 & 0.19 & 0.01 & $-0.32 * *$ \\
\hline 1-back & $0.35^{* *}$ & $0.25^{*}$ & 0.03 & -0.16 \\
\hline 2-back & 0.22 & 0.20 & 0.09 & -0.07 \\
\hline \multicolumn{5}{|c|}{ COGNITIVE DOMAINS } \\
\hline IO & 0.18 & $0.24^{*}$ & 0.11 & -0.03 \\
\hline Working memory & $0.32 * *$ & 0.19 & $0.28^{*}$ & 0.09 \\
\hline Episodic memory & $0.33^{* *}$ & 0.19 & 0.20 & 0.15 \\
\hline Executive function & $0.34^{* *}$ & $0.30^{*}$ & 0.17 & 0.04 \\
\hline \multicolumn{5}{|l|}{ CLINICAL DOMAINS } \\
\hline Positive symptoms & 0.01 & -0.13 & -0.09 & -0.08 \\
\hline Negative symptoms & -0.20 & -0.15 & -0.07 & -0.02 \\
\hline Disorganization & $-0.26^{*}$ & $-0.25^{*}$ & 0.01 & -0.08 \\
\hline
\end{tabular}

${ }^{*} p<0.05 ;{ }^{*} p<0.01$.

shown in Figure S10 in Supplementary Material the relationship between bFP-CER and disorganization symptoms is being driven by the SCZ, who have the most variance. Resting state bFP-CER (see Figure S8 in Supplementary Material) also predicted better performance on the $N$-back task (1-back accuracy), a result that was significant even among the SCZ and SCZ-SIB individually, but with similar trends in the CON and CON-SIB. In contrast, the task connectivity measures were not nearly so consistently associated with the cognitive variables, and were not associated with the clinical variables. Stronger task bFP-CER connectivity was not associated with better working memory performance. However, stronger task bCO-CER connectivity was actually associated with worse 0-back performance. We also present scatterplots for these correlations in Figures S8-S10 in Supplementary Material.

\section{DISCUSSION}

The goal of the current study was to examine the degree to which changes in functional connectivity in schizophrenia were dependent on the cognitive state of the individual (rest versus during working memory task performance) as a means to shed light on the potential mechanisms leading to altered functional connectivity in this illness. As a brief summary, we found that connectivity within the DMN and FP increased significantly between resting state and 0-back, while connectivity within the CO and CER decreased significantly between resting state and 0-back. Further, the DMN became significantly more "anti-correlated" with the FP, CO, and CER networks during 0-back as compared to rest. Additionally, we found that connectivity within both the DMN and FP was further modulated by memory load, and that connectivity between the FP and both CO and CER networks increased with memory load. Individuals with schizophrenia and their siblings showed consistent reductions in connectivity between both the FP and CO networks with the CER network, a finding that was similar in magnitude across rest and all levels of working memory load. The latter results are consistent with the hypothesis that functional connectivity changes associated with genetic liability to schizophrenia reflect stable alterations in brain connectivity that are not dependent on the state of the individual. We discuss each of these findings in more detail below.

\section{CONNECTIVITY CHANGES AS A FUNCTION OF TASK STATE AND MEMORY LOAD}

Consistent with prior work, we found that connectivity both within and between networks changed as a function of task state and working memory load (see Figure 6 for a summary). Specifically, we found that connectivity within the FP increased during 0 -back compared to rest, and continued to increase as a function of load. These findings are consistent with prior studies showing such connectivity increases among regions involved in the FP during working memory (Honey et al., 2002; Woodward et al., 2006; Ma et al., 2011; Nagel et al., 2011; Newton et al., 2011). The pattern of connectivity changes within the DMN was more complicated. Connectivity increased from rest to 0-back, generally consistent with the finding of Newton et al. (2011). However, connectivity then decreased again at 1-back, and even further at 2-back. Newton also found that DMN connectivity decreased at the highest working memory loads (though this was 3-back) and other researchers have found significant decreases in connectivity within the DMN during 2-back as compared to rest (Fransson, 2006). Thus, it is clear that DMN connectivity is modulated by task engagement and working memory load, but the exact pattern is more variable across studies than in the FP. In contrast to FP and DMN, connectivity within the $\mathrm{CO}$ and the CER networks decreased from rest to 0-back, but did not show any further load modulation. Such results suggest that connectivity within the $\mathrm{CO}$ and CER networks may dynamically reorganize as a function of overall task engagement, but not based on changes in difficulty within the task (e.g., load). This interpretation is broadly consistent with the suggestion that the CO network is involved in stable task set maintenance 


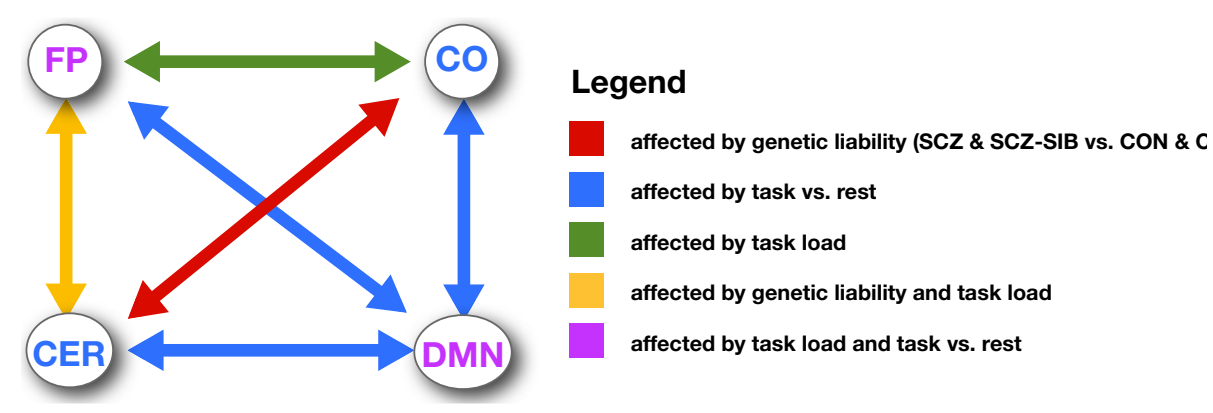

FIGURE 6 | Figure illustrating the pattern of changes in connectivity both within and between networks as a function of task state, memory load, and genetic liability.

(Dosenbach et al., 2006, 2007, 2008), while the FP may be more involved in dynamic modulation of task sets.

Interestingly, connectivity between the DMN and all three other networks became more negative in the 0 -back conditions as compared to rest, and stayed stable across memory loads for DMN connectivity to FP and CER. However, DMN to CO connectivity became more similar to rest with increased memory load. The findings for DMN connectivity to FB and CER are consistent with prior work showing that at least parts of the DMN become more "anti-correlated" with the task-positive network during working memory performance (Leech et al., 2011). Further, such findings are consistent with the argument that effective task performance is associated with decreased activity in DMN regions as means of suppressing "off-task" cognitions (Shulman et al., 2003; Anticevic et al., 2010). However, other researchers have not always found significant increases in DMN anti-correlation with task-positive networks during task versus rest (Hampson et al., 2006; Newton et al., 2011), though this reflect power issues given the relatively small sample sizes in these prior studies.

Connectivity between the FP, CO, and CER networks did not change significantly between rest and 0 -back. However, connectivity between the FP and both the CO and CER networks increased significantly as a function of memory load. The increased connectivity between FP and CO is consistent with several prior studies (Woodward et al., 2006; Nagel et al., 2011) and with the role of FP in dynamic task set modulation (Dosenbach et al., 2006). Further, it is consistent with the role of the $\mathrm{CO}$ in responding to errors and task conflict that may increase as a function of memory load (Botvinick et al., 2001, 2004). The increased connectivity between FP and CER as a function of memory load is also consistent with the fact that errors increase as a function of memory load, perhaps reflecting an enhanced need for error processing mechanisms supported by the CER (Ide and Li, 2011), which may signal the need for increased control provided by the FP network.

\section{CONNECTIVITY CHANGES ASSOCIATED WITH GENETIC LIABILITY TO SCHIZOPHRENIA AS A FUNCTION OF TASK STATE AND MEMORY LOAD}

We found very consistent evidence across task states and memory loads for altered connectivity in individuals with schizophrenia and their siblings, with reductions between the FP and $\mathrm{CO}$ networks and the CER network (see Figure 6). These connectivity reductions were present both at rest and across all working memory loads. The resting state findings are not new, and were the focus of a previous report (Repovs et al., 2011). However, the presence of similar changes in connectivity during working memory is a novel finding. Importantly, the magnitude of these connectivity reductions did not change as a function of task state. Further, individuals with schizophrenia and their siblings still showed significant increases in connectivity between FP and CER as a function of memory load, despite an overall reduction in connectivity. Additionally, the individuals with schizophrenia and their siblings also showed similar changes in connectivity between the FP and CO networks, as well as between the DMN and CO networks and within both the DMN and FP networks, as a function of memory load (though the genetic liability effects involving the DMN would not pass FDR correction). This pattern of results suggests two important things about the source of connectivity changes in schizophrenia. First, they suggest that these connectivity changes in schizophrenia patients and their siblings are unlikely to be due to confounding factors such as differences in what patients are thinking about during rest state scans, as the imposition of a structured task state did not alter the pattern of connectivity changes. Second, the fact that patients and their siblings showed a relatively intact ability to modulate connectivity as a function of task demands suggest that these connectivity changes are not simply the results of decreased task engagement. Instead, this pattern of results is more consistent with connectivity changes reflecting a more fundamental and/or trait like change in brain connectivity. Third, the fact that similar changes were seen in the individuals with schizophrenia and their siblings suggests that the results are likely more indicative of genetic liability to schizophrenia, rather than manifest disease itself. Functional connectivity is not isomorphic with structural connectivity (at least in terms of single synapse connections) and thus one cannot directly interpret alterations in function connectivity as reflecting alterations in structural connectivity. However, findings such as these point to the need to more directly examine the degree to which changes in functional connectivity are reflective of changes in white matter integrity and connections in schizophrenia. A growing number of studies have started to address this question, finding important initial evidence for overlap and interrelations between structural and functional connectivity changes in schizophrenia (Liu et al., 2008, 2011; Pomarol-Clotet et al., 2010; Camchong et al., 2011), and this is clearly an area ripe for additional research. 
We also examined the relationship between individual differences in wDMN, bFP-CER, and bCO-CER connectivity and symptoms, neuropsychological performance and working memory task performance during fMRI scanning. Connectivity between $\mathrm{CO}$ and CER and connectivity within the DMN network did not predict cognitive performance or symptoms. However, as found in our prior work in this sample (Repovs et al., 2011), greater connectivity between the FP and CER networks predicted better performance on neuropsychological measures of working memory, episodic memory, and executive function, as well as fewer disorganization symptoms. Importantly, we also found that greater FP to CER connectivity predicted better accuracy on the $N$-back task (1back condition) performed during fMRI scanning. Interestingly, however, these relationships were only significant for resting state connectivity and not for task connectivity. This was somewhat surprising to us, as we would have predicted greater relationships for task as compared to resting state connectivity. For the neuropsychological measures, the general patterns were the same for task and resting state connectivity, but this was not true for $N$-back performance or symptoms. Such findings could indicate that resting state data is a more sensitive indicator of functional connectivity changes relevant for cognitive performance and symptom manifestation, but such results need to be replicated in order to be confident in such an interpretation.

Of note, the primary analyses of within-network connectivity as a function of either task or memory load did not provide robust evidence for changes in individuals with schizophrenia or their siblings. However, the follow-up contrasts did reveal some evidence for altered connectivity within the DMN among individuals with schizophrenia, with less modulation of DMN as a function of task state and memory load. Although not robust, these findings are consistent with a number of other studies suggesting reduced DMN activity in schizophrenia during rest (Bluhm et al., 2007, 2009; Ongur et al., 2010; Rotarska-Jagiela et al., 2010; Camchong et al., 2011) and during task (Garrity et al., 2007; Kim et al., 2009a,b; Meda et al., 2009), though other studies have also found increased DMN activity in this illness (Whitfield-Gabrieli et al., 2009) or no differences in DMN connectivity (Woodward et al., 2011). These findings suggest that the exact patterns of DMN connectivity changes in schizophrenia are quite variable, and may be dependent both on task and sample characteristics, factors that need to be systematically examined in future studies.

It is of further interest to note that we found the most consistent connectivity changes in individuals with schizophrenia and their siblings in connections involving the CER, and the most consistent individual difference relationships were with the magnitude of connectivity between the FP and CER networks. These results are consistent with previous suggestions that cognitive impairments in schizophrenia reflect deficits in cortical-subcortical-cerebellar circuits (Andreasen et al., 1998; Andreasen and Pierson, 2008). There has been increasing interest in the cognitive and affective processing functions of the CER in recent years (Stoodley and Schmahmann, 2010; Koziol et al., 2011; O'Halloran et al., 2012), but it is not yet clear exactly how the CER contributes higher level cognition. One speculation is that the CER may play a key role in learning from errors, and in the timing and sequencing of a range of cognitive functions (Fiez et al., 1992; Fiez, 1996; Ravizza et al.,
2006; Ben-Yehudah et al., 2007; Strick et al., 2009; Durisko and Fiez, 2010). Thus, disruptions in the coordination of CER activity with other networks may have major implications for impairments in cognitive adaptation and coordination, and may be relevant for understanding genetic liability to schizophrenia.

\section{LIMITATIONS}

There were several limitations in the current study. First, all of the individuals with schizophrenia were taking antipsychotic medication, and some prior research has suggested that medications may alter connectivity in schizophrenia (Lui et al., 2010). However, we found similar results in the siblings of individuals with schizophrenia, and none of the siblings were taking antipsychotic medications. This makes it unlikely that our primary findings were artifacts of medication status. Second, our prior study on resting state connectivity also found reductions in FP to CO connectivity among individuals with schizophrenia and their siblings. We did not find such changes in the current study, although the pattern was in the same direction in all conditions (lower connectivity in SCZ and SCZ-SIB). This could reflect the fact that some participants included in the prior study were not included in the current study, as they did not have task connectivity data. Alternatively, it could reflect the more stringent movement correction procedures implemented in the current study, based on the recently published work of Power and Petersen (Power et al., 2011). We also saw some trend level genetic liability effects involving the DMN, which did not pass FDR correction. It is possible this reflected power issues. With our sample size, we have $67 \%$ power to detect a medium effect size for the main effect of genetic liability in the task versus rest analysis, and $74 \%$ power in the working memory load analyses. At minimum however, our results suggest that the genetic liability effects on bFP-CER and bCO-CER connectivity changes were stronger than any such effects on connectivity involving DMN. One might also be concerned that including the siblings of individuals with schizophrenia and looking for main effects of genetic liability or interactions between genetic liability and family member type might have reduced power. To address this concern, we repeated all analyses with just the healthy controls and their siblings (treated as a single control group) and individuals with schizophrenia, excluding the siblings of the individuals with schizophrenia. As reported in the Supplementary Material, these analyses provided essentially identical results to the main analyses, suggesting that the inclusion of the siblings of individuals with schizophrenia did not mask any significant effects in the individuals with schizophrenia and that the inclusions of individual who shared some genetic relationship did not create spurious statistical results.

\section{CONCLUSION}

In summary, the current study provided robust evidence for reduced connectivity between the FP and CER networks and the CO and CER networks among individuals with schizophrenia and their siblings. These changes were present both at rest and during working memory task performance, and the magnitude of group differences in connectivity did not change as a function of task state or memory load. Such findings suggest that connectivity changes between networks involved in both 
dynamic and stable task control and error processing in schizophrenia reflect fundamental changes in brain connectivity that are not secondary to task engagement or other state related factors, and which may reflect genetic liability to the illness. Further, changes in FP to CER connectivity predicted neuropsychological performance, symptoms, and $N$-back performance, though these relationships were stronger for resting state than task-based connectivity. These findings point to the need to examine the influence of changes in white matter integrity on alterations in functional connectivity, as a means to understand the causes of these robust changes in functional connectivity in schizophrenia that cut across task states and are present both in individuals with manifest illness and those genetically at risk for schizophrenia.

\section{REFERENCES}

Andreasen, N. C., Paradiso, S., and O'Leary, D. S. (1998). "Cognitive dysmetria" as an integrative theory of schizophrenia: a dysfunction in cortical-subcorticalcerebellar circuitry? Schizophr. Bull. 24, 203-218.

Andreasen, N. C., and Pierson, R. (2008). The role of the cerebellum in schizophrenia. Biol. Psychiatry 64, 81-88.

Anticevic, A., Repovs, G., and Barch, D. M. (2011). Emotion effects on attention, amygdala activation, and functional connectivity in schizophrenia. Schizophr. Bull. doi: 10.1093/schbul/sbq168

Anticevic, A., Repovs, G., Shulman, G. L., and Barch, D. M. (2010). When less is more: TPJ and default network deactivation during encoding predicts working memory performance. Neuroimage 49, 2638-2648.

Barch, D. M., and Csernansky, J. G. (2007). Abnormal parietal cortex activation during working memory in schizophrenia: verbal phonological coding disturbances versus domain-general executive dysfunction. Am. J. Psychiatry 164, 1090-1098.

Bassett, D. S., Bullmore, E., Verchinski, B. A., Mattay, V. S., Weinberger, D. R., and Meyer-Lindenberg, A. (2008). Hierarchical organization of human cortical networks in health and schizophrenia. J. Neurosci. 28, 9239-9248.

Bassett, D. S., Nelson, B. G., Mueller, B. A., Camchong, J., and Lim, K. O. (2011). Altered resting state complexity in schizophrenia. Neuroimage 59, 2196-2207.

Becerril, K. E., Repovs, G., and Barch, D. M. (2011). Error processing network dynamics in schizophrenia. Neuroimage 54, 1495-1505.

Ben-Yehudah, G., Guediche, S., and Fiez, J. A. (2007). Cerebellar contributions to verbal working memory: beyond cognitive theory. Cerebellum 6, 193-201.

Bluhm, R. L., Miller, J., Lanius, R. A., Osuch, E. A., Boksman, K., Neufeld, R. W., Theberge, J., Schaefer, B., and Williamson, P. (2007). Spontaneous low-frequency fluctuations in the BOLD signal in schizophrenic patients: anomalies in the default network. Schizophr. Bull. 33, 1004-1012.

Bluhm, R. L., Miller, J., Lanius, R. A., Osuch, E. A., Boksman, K., Neufeld, R. W., Theberge, J., Schaefer, B., and Williamson, P. C. (2009). Retrosplenial cortex connectivity in schizophrenia. Psychiatry Res. 174, 17-23.

Botvinick, M. M., Braver, T. S., Barch, D. M., Carter, C. S., and Cohen, J. C. (2001). Conflict monitoring and cognitive control. Psychol. Rev. 108, 624-652.

Botvinick, M. M., Cohen, J. D., and Carter, C. S. (2004). Conflict monitoring and anterior cingulate cortex: an update. Trends Cogn. Sci. (Regul. Ed.) 8, 539-546.

Boynton, G. M., Engel, S. A., Glover, G. H., and Heeger, D. J. (1996). Linear systems analysis of functional magnetic resonance imaging in human V1. J. Neurosci. 16, 4207-4221.

Brown, G. G., and Thompson, W. K. (2010). Functional brain imaging in schizophrenia: selected results and methods. Curr. Top. Behav. Neurosci. 4, 181-214.

Calhoun, V. D., Eichele, T., and Pearlson, G. (2009). Functional brain networks in schizophrenia: a review. Front. Hum. Neurosci. 3:17. doi:10.3389/neuro.09.017.2009

Camchong, J., MacDonald, A. W. III, Bell, C., Mueller, B. A., and Lim, K. O. (2011). Altered functional and anatomical connectivity in schizophrenia. Schizophr. Bull.37, 640-650.

\section{ACKNOWLEDGMENTS}

We thank the staff of the Administrative/Assessment and Biostatistical Cores of the CCNMD at Washington University School of Medicine for collection of the clinical and imaging data and data management. This research was supported by NIH grants P50 MH071616 and R01 MH56584. Financial Disclosures: Dr. Repovš is a consultant on NIMH grants. Dr. Barch has received grants from the NIMH, NIA, NARSAD, Allon, Novartis, and the McDonnell Center for Systems Neuroscience and has consulted for Pfizer.

\section{SUPPLEMENTARY MATERIAL}

The Supplementary Material for this article can be found online at: http://www.frontiersin.org/Human_Neuroscience/10.3389/

fnhum.2012.00137/abstract

Chai, X. J., Whitfield-Gabrieli, S., Shinn, A. K., Gabrieli, J. D., Nieto Castanon, A., McCarthy, J. M., Cohen, B. M., and Ongur, D. (2011). Abnormal medial prefrontal cortex restingstate connectivity in bipolar disorder and schizophrenia. Neuropsychopharmacology 36, 2009-2017.

Cole, M. W., Anticevic, A., Repovs, G. and Barch, D. (2011). Variable global dysconnectivity and individual differences in schizophrenia. Biol. Psychiatry 70, 43-50.

Crossley, N. A., Mechelli, A., Fusar-Poli, P., Broome, M. R., Matthiasson, P., Johns, L. C., Bramon, E., Valmaggia, L., Williams, S. C., and McGuire, P. K. (2009). Superior temporal lobe dysfunction and frontotemporal dysconnectivity in subjects at risk of psychosis and in first-episode psychosis. Hum. Brain Mapp. 30, 4129-4137.

Damoiseaux, J. S., Rombouts, S. A., Barkhof, F., Scheltens, P., Stam, C. J., Smith, S. M., and Beckmann, C. F. (2006). Consistent resting-state networks across healthy subjects. Proc. Natl. Acad. Sci. U.S.A. 103, 13848-13853.

Delawalla, Z., Barch, D. M., Fisher Eastep, J. L., Thomason, E. S., Hanewinkel, M. J., Thompson, P. A., and Csernansky, J. G. (2006). Factors mediating cognitive deficits and psychopathology among siblings of individuals with schizophrenia. Schizophr. Bull. 32, 525-537.

Diaconescu, A. O., Jensen, J., Wang, H., Willeit, M., Menon, M., Kapur, S., and McIntosh, A. R. (2011). Aberrant effective connectivity in schizophrenia patients during appetitive conditioning. Front. Hum. Neurosci. 4:239. doi:10.3389/fnhum.2010.00239

Dosenbach, N. U., Fair, D. A., Cohen, A. L., Schlaggar, B. L., and Petersen, S. E. (2008). A dual-networks architecture of top-down control.
Trends Cogn. Sci. (Regul. Ed.). 12, 99-105.

Dosenbach, N. U., Fair, D. A., Miezin, F. M., Cohen, A. L., Wenger, K. K., Dosenbach, R. A., Fox, M. D., Snyder, A. Z., Vincent, J. L., Raichle, M. E., Schlaggar, B. L., and Petersen, S. E. (2007). Distinct brain networks for adaptive and stable task control in humans. Proc. Natl. Acad. Sci. U.S.A. 104, 11073-11078.

Dosenbach, N. U., Nardos, B., Cohen, A. L., Fair, D. A., Power, J. D., Church, J. A., Nelson, S. M., Wig, G. S., Vogel, A. C., Lessov-Schlaggar, C. N., Barnes, K. A., Dubis, J. W., Feczko, E., Coalson, R. S., Pruett, J. R. Jr., Barch, D. M., Petersen, S. E., and Schlaggar, B. L. (2010). Prediction of individual brain maturity using fMRI. Science 329, 1358-1361.

Dosenbach, N. U., Visscher, K. M., Palmer, E. D., Miezin, F. M., Wenger, K. K., Kang, H. C., Burgund, E. D., Grimes, A. L., Schlaggar, B. L., and Petersen, S. E. (2006). A core system for the implementation of task sets. Neuron 50, 799-812.

Durisko, C., and Fiez, J. A. (2010). Functional activation in the cerebellum during working memory and simple speech tasks. Cortex 46, 896-906.

Fair, D. A., Dosenbach, N. U., Church, J. A., Cohen, A. L., Brahmbhatt, S., Miezin, F. M., Barch, D. M., Raichle, M. E., Petersen, S. E., and Schlaggar, B. L. (2007a). Development of distinct control networks through segregation and integration. Proc. Natl. Acad. Sci. U.S.A. 104, 13507-13512.

Fair, D. A., Dosenbach, N. U., Church, J. A., Cohen, A. L., Brahmbhatt, S., Miezin, F. M., Barch, D. M., Raichle, M. E., Petersen, S. E., and Schlaggar, B. L. (2007b). Development of distinct control networks through segregation and integration. Proc. Natl. Acad. Sci. U.S.A. 104, 13507-13512. 
Fair, D. A., Schlaggar, B. L., Cohen, A. L., Miezin, F. M., Dosenbach, N. U., Wenger, K. K., Fox, M. D., Snyder, A. Z., Raichle, M. E., and Petersen, S. E. $(2007 \mathrm{c})$. A method for using blocked and event-related fMRI data to study "resting state" functional connectivity. Neuroimage 35, 396-405.

Fiez, J. A. (1996). Cerebellar contributions to cognition. Neuron 16, 13-15.

Fiez, J. A., Petersen, S. E., Cheney, M. K., and Raichle, M. E. (1992). Impaired non-motor learning and error detection associated with cerebellar damage. A single case study. Brain 115 (Pt 1), 155-178

First, M. B., Spitzer, R. L., Gibbon, M., and Williams, J. B. W. (2001). Structured Clinical Interview for the DSMIV-TR Axis I Disorders. Washington, DC: American Psychiatric Press.

Fischl, B., Salat, D. H., Busa, E., Albert, M., Dieterich, M., Haselgrove, C., Van Der Kouwe, A., Killiany, R., Kennedy, D., Klaveness, S., Montillo, A., Makris, N., Rosen, B., and Dale, A. M. (2002). Whole brain segmentation: automated labeling of neuroanatomical structures in the human brain. Neuron 33, 341-355.

Forbes, N. F., Carrick, L. A., McIntosh, A. M., and Lawrie, S. M. (2009). Working memory in schizophrenia: a meta-analysis. Psychol. Med. 39, 889-905.

Fornito, A., Yoon, J., Zalesky, A., Bullmore, E. T., and Carter, C. S. (2011). General and specific functional connectivity disturbances in first-episode schizophrenia during cognitive control performance. Biol. Psychiatry 70, 64-72.

Fox, M. D., Snyder, A. Z., Vincent, J. L., Corbetta, M., Van Essen, D. C., and Raichle, M. E. (2005). The human brain is intrinsically organized into dynamic, anticorrelated functional networks. Proc. Natl. Acad. Sci. U.S.A. 102, 9673-9678.

Fransson, P. (2006). How default is the default mode of brain function? Further evidence from intrinsic BOLD signal fluctuations. Neuropsychologia 44, 2836-2845.

Garrity, A. G., Pearlson, G. D., McKiernan, K., Lloyd, D., Kiehl, K. A., and Calhoun, V. D. (2007). Aberrant "default mode" functional connectivity in schizophrenia. Am. J. Psychiatry 164, 450-457.

Hampson, M., Driesen, N. R., Skudlarski, P., Gore, J. C., and Constable, R. T. (2006). Brain connectivity related to working memory performance. J. Neurosci. 26, 13338-13343.

Harms, M. P., Wang, L., Mamah, D., Barch, D. M., Thompson, P. A., and Csernansky, J. G. (2007). Thalamic shape abnormalities in individuals with schizophrenia and their nonpsychotic siblings. J. Neurosci. 27, 13835-13842.

Henseler, I., Falkai, P., and Gruber, O. (2010). Disturbed functional connectivity within brain networks subserving domain-specific subcomponents of working memory in schizophrenia: relation to performance and clinical symptoms. J. Psychiatr. Res. 44, 364-372.

Honey, G. D., Fu, C. H., Kim, J., Brammer, M. J., Croudace, T. J., Suckling, J., Pich, E. M., Williams, S. C., and Bullmore, E. T. (2002). Effects of verbal working memory load on corticocortical connectivity modeled by path analysis of functional magnetic resonance imaging data. Neuroimage $17,573-582$.

Ide, J. S., and Li, C. S. (2011). A cerebellar thalamic cortical circuit for error-related cognitive control. $\mathrm{Neu}$ roimage 54, 455-464.

Jafri, M. J., Pearlson, G. D., Stevens, M., and Calhoun, V. D. (2008). A method for functional network connectivity among spatially independent resting-state components in schizophrenia. Neuroimage 39, 1666-1681.

Kang, S. S., Sponheim, S. R., Chafee, M. V., and MacDonald, A. W. III. (2011). Disrupted functional connectivity for controlled visual processing as a basis for impaired spatial working memory in schizophrenia. Neuropsychologia 49, 2836-2847.

Kim, D. I., Manoach, D. S., Mathalon, D. H., Turner, J. A., Mannell, M., Brown, G. G., Ford, J. M., Gollub, R. L., White, T., Wible, C., Belger, A., Bockholt, H. J., Clark, V. P., Lauriello, J., O’Leary, D., Mueller, B. A., Lim, K. O., Andreasen, N., Potkin, S. G., and Calhoun, V. D. (2009a). Dysregulation of working memory and default-mode networks in schizophrenia using independent component analysis, an fBIRN and MCIC study. Hum. Brain Mapp. 30, 37953811.

Kim, D. I., Mathalon, D. H., Ford, J. M., Mannell, M., Turner, J. A., Brown, G. G., Belger, A., Gollub, R., Lauriello, J., Wible, C., O’Leary, D., Lim, K., Toga, A., Potkin, S. G., Birn, F., and Calhoun, V. D. (2009b). Auditory oddball deficits in schizophrenia: an independent component analysis of the fMRI multisite function BIRN study. Schizophr. Bull. 35, 67-81.

Koziol, L. F., Budding, D. E., and Chidekel, D. (2011). From movement to thought: executive function, embodied cognition, and the cerebellum. Cerebellum. doi: 10.1007/s12311-011-0321-y. [Epub ahead of print]

Leech, R., Kamourieh, S., Beckmann, C. F., and Sharp, D. J. (2011). Fractionating the default mode network: distinct contributions of the ventral and dorsal posterior cingulate cortex to cognitive control. J. Neurosci. 31, 3217-3224

Liu, H., Fan, G., Xu, K., and Wang, F. (2011). Changes in cerebellar functional connectivity and anatomical connectivity in schizophrenia: a combined resting-state functional MRI and diffusion tensor imaging study. J. Magn. Reson. Imaging 34, 1430-1438.

Liu, Y., Liang, M., Zhou, Y., He, Y., Hao, Y., Song, M., Yu, C., Liu, H., Liu, Z., and Jiang, T. (2008). Disrupted small-world networks in schizophrenia. Brain 131, 945-961.

Lui, S., Li, T., Deng, W., Jiang, L., Wu, Q. Tang, H., Yue, Q., Huang, X., Chan, R. C., Collier, D. A., Meda, S. A., Pearlson, G., Mechelli, A., Sweeney, J. A., and Gong, Q. (2010). Short-term effects of antipsychotic treatment on cerebral function in drug-naive first-episode schizophrenia revealed by "resting state" functional magnetic resonance imaging. Arch. Gen. Psychiatry 67, 783-792.

Lynall, M. E., Bassett, D. S., Kerwin, R., McKenna, P. J., Kitzbichler, M., Muller, U., and Bullmore, E. (2010). Functional connectivity and brain networks in schizophrenia. J. Neurosci. 30, 9477-9487.

Ma, L., Steinberg, J. L., Hasan, K. M., Narayana, P. A., Kramer, L. A., and Moeller, F. G. (2011). Working memory load modulation of parieto-frontal connections: evidence from dynamic causal modeling. Hum. Brain Mapp. doi: 10.1002/hbm.21329. [Epub ahead of print].

Mannell, M. V., Franco, A. R., Calhoun, V. D., Canive, J. M., Thoma, R. J., and Mayer, A. R. (2010). Resting state and task-induced deactivation: a methodological comparison in patients with schizophrenia and healthy controls. Hum. Brain Mapp. 31, 424-437.

Meda, S. A., Stevens, M. C., Folley, B. S., Calhoun, V. D., and Pearlson, G. D. (2009). Evidence for anomalous network connectivity during working memory encoding in schizophrenia: an ICA based analysis. PLoS ONE 4, e7911. doi:10.1371/journal.pone.0007911

Meyer-Lindenberg, A., Poline, J., Kohn, P. D., Holt, J. L., Egan, M. F., Weinberger, D. R., and Berman, K. F.
(2001). Evidence for abnormal cortical functional connectivity during working memory in schizophrenia. Am. J. Psychiatry 158, 1809-1817.

Meyer-Lindenberg, A. S., Olsen, R. K., Kohn, P. D., Brown, T., Egan, M. F., Weinberger, D. R., and Berman, K. F. (2005). Regionally specific disturbance of dorsolateral prefrontalhippocampal functional connectivity in schizophrenia. Arch. Gen. Psychiatry 62, 379-386.

Micheloyannis, S., Pachou, E., Stam, C. J., Breakspear, M., Bitsios, P., Vourkas, M., Erimaki, S., and Zervakis, M. (2006). Small-world networks and disturbed functional connectivity in schizophrenia. Schizophr. Res. 87, 60-66.

Mukherjee, P., Whalley, H. C., McKirdy, J. W., McIntosh, A. M., Johnstone, E. C., Lawrie, S. M., and Hall, J. (2011). Lower effective connectivity between amygdala and parietal regions in response to fearful faces in schizophrenia. Schizophr. Res. 134, 118-124.

Nagel, I. E., Preuschhof, C., Li, S. C., Nyberg, L., Backman, L., Lindenberger, U., and Heekeren, H. R. (2011). Load modulation of BOLD response and connectivity predicts working memory performance in younger and older adults. J. Cogn. Neurosci. 23, 2030-2045.

Newton, A. T., Morgan, V. L., Rogers, B. P., and Gore, J. C. (2011). Modulation of steady state functional connectivity in the default mode and working memory networks by cognitive load. Hum. Brain Mapp. 32, 1649-1659.

O'Halloran, C. J., Kinsella, G. J., and Storey, E. (2012). The cerebellum and neuropsychological functioning: a critical review. J. Clin. Exp. Neuropsychol. 34, 35-56.

Ojemann, J., Akbudak, E., Snyder, A., McKinstry, R., Raichle, M., and Conturo, T. (1997). Anatomic localization and quantitative analysis of gradient refocused echo-planar fMRI susceptibility artifacts. Neuroimage 6, 156-167.

Ongur, D., Lundy, M., Greenhouse, I., Shinn, A. K., Menon, V., Cohen, B. M., and Renshaw, P. F. (2010). Default mode network abnormalities in bipolar disorder and schizophrenia. Psychiatry Res. 183, 59-68.

Pomarol-Clotet, E., Canales-Rodriguez, E. J., Salvador, R., Sarro, S., Gomar, J. J., Vila, F., Ortiz-Gil, J., IturriaMedina, Y., Capdevila, A., and McKenna, P. J. (2010). Medial prefrontal cortex pathology in schizophrenia as revealed by convergent 
findings from multimodal imaging. Mol. Psychiatry 15, 823-830.

Power, J. D., Barnes, K. A., Snyder, A. Z., Schlaggar, B. L., and Petersen, S. E. (2011). Spurious but systematic correlations in functional connectivity MRI networks arise from subject motion. Neuroimage 59, 2142-2154.

Raichle, M. E., and Snyder, A. Z. (2007). A default mode of brain function: a brief history of an evolving idea. Neuroimage 37, 1083-1090.

Rasetti, R., Sambataro, F., Chen, Q., Callicott, J. H., Mattay, V. S., and Weinberger, D. R. (2011). Altered cortical network dynamics: a potential intermediate phenotype for schizophrenia and association with ZNF804A. Arch. Gen. Psychiatry 68, 1207-1217.

Ravizza, S. M., McCormick, C. A., Schlerf, J. E., Justus, T., Ivry, R. B., and Fiez, J. A. (2006). Cerebellar damage produces selective deficits in verbal working memory. Brain 129, 306-320.

Repovs, G., Csernansky, J. G., and Barch, D. M. (2011). Brain network connectivity in individuals with schizophrenia and their siblings. Biol. Psychiatry 69, 967-973.

Rotarska-Jagiela, A., Van De Ven, V., Oertel-Knochel, V., Uhlhaas, P. J., Vogeley, K., and Linden, D. E. (2010). Resting-state functional network correlates of psychotic symptoms in schizophrenia. Schizophr. Res. 117, 21-30.

Salomon, R., Bleich-Cohen, M., Hahamy-Dubossarsky, A., Dinstien, I., Weizman, R., Poyurovsky, M., Kupchik, M., Kotler, M., Hendler, T., and Malach, R. (2011). Global functional connectivity deficits in schizophrenia depend on behavioral state. J. Neurosci. 31, 12972-12981.

Salvador, R., Sarro, S., Gomar, J. J., Ortiz-Gil, J., Vila, F., Capdevila, A., Bullmore, E., McKenna, P. J., and Pomarol-Clotet, E. (2010). Overall brain connectivity maps show cortico-subcortical abnormalities in schizophrenia. Hum. Brain Mapp. 31, 2003-2014.

Schlosser, R., Gesierich, T., Kaufmann, B., Vucurevic, G., Hunsche, S., Gawehn, J., and Stoeter, P. (2003a). Altered effective connectivity during working memory performance in schizophrenia: a study with fMRI and structural equation modeling. Neuroimage 19, 751-763.

Schlosser, R., Gesierich, T., Kaufmann, B., Vucurevic, G., and Stoeter, P. (2003b). Altered effective connectivity in drug free schizophrenic patients. Neuroreport 14, 2233-2237.

Shulman, G. L., McAvoy, M. P., Cowan, M. C., Astafiev, S. V., Tansy, A. P., D'Avossa, G., and Corbetta, M. (2003). Quantitative analysis of attention and detection signals during visual search. J. Neurophysiol. 90, 3384-3397.

Stephan, K. E., Friston, K. J., and Frith, C. D. (2009). Dysconnection in schizophrenia: from abnormal synaptic plasticity to failures of self-monitoring. Schizophr. Bull. 35, 509-527.

Stoodley, C. J., and Schmahmann, J. D. (2010). Evidence for topographic organization in the cerebellum of motor control versus cognitive and affective processing. Cortex 46, 831-844.

Strick, P. L., Dum, R. P., and Fiez, J. A. (2009). Cerebellum and nonmotor function. Annu. Rev. Neurosci. 32, 413-434.

Sutton, S. (1973). "Fact and artifact in the psychology of schizophrenia," in Psychopathology: Contributions from the Social, Behavioral and Biological Sciences, eds M. Hammer, K. Slakinger, and S. Sutton (New York: John Wiley and Sons), 197-213.

Talairach, J., and Tournoux, P. (1988). Co-Planar Stereotaxic Atlas of the Human Brain. New York: Thieme.

Team, R. D. C. (2011). A Language and Environment for Statistical Computing. Vienna: Foundation for Statistical Computing.
Tu, P. C., Hsieh, J. C., Li, C. T., Bai, Y. M., and Su, T. P. (2012). Corticostriatal disconnection within the cingulo-opercular network in schizophrenia revealed by intrinsic functional connectivity analysis: a resting fMRI study. Neuroimage 59, 238-247.

Welsh, R. C., Chen, A. C., and Taylor, S. F. (2008). Low-frequency BOLD fluctuations demonstrate altered thalamocortical connectivity in schizophrenia. Schizophr. Bull.

White, T., Schmidt, M., Kim, D. I. and Calhoun, V. D. (2011). Disrupted functional brain connectivity during verbal working memory in children and adolescents with schizophrenia. Cereb. Cortex 21, 510-518.

Whitfield-Gabrieli, S., Thermenos, $\mathrm{H}$. W., Milanovic, S., Tsuang, M. T. Faraone, S. V., McCarley, R. W., Shenton, M. E., Green, A. I., NietoCastanon, A., Laviolette, P., Wojcik, J., Gabrieli, J. D., and Seidman, L. J. (2009). Hyperactivity and hyperconnectivity of the default network in schizophrenia and in first-degree relatives of persons with schizophrenia. Proc. Natl. Acad. Sci. U.S.A. 106 1279-1284.

Wickham, H. (2009). Elegant Graphics for Data Analysis. New York: Springer.

Wolf, R. C., Vasic, N., Sambataro, F., Hose, A., Frasch, K., Schmid, M., and Walter, H. (2009). Temporally anticorrelated brain networks during working memory performance reveal aberrant prefrontal and hippocampal connectivity in patients with schizophrenia. Prog. Neuropsychopharmacol. Biol. Psychiatry 33, 1464-1473.

Woodward, N. D., Rogers, B., and Heckers, S. (2011). Functional restingstate networks are differentially affected in schizophrenia. Schizophr. Res. 130, 86-93.

Woodward, T. S., Cairo, T. A., Ruff, C. C., Takane, Y., Hunter, M. A., and Ngan, E. T. (2006). Functional connectivity reveals load dependent neural systems underlying encoding and maintenance in verbal working memory. Neuroscience 139, 317-325.

Yu, Q., Sui, J., Rachakonda, S., He, H., Pearlson, G., and Calhoun, V. D. (2011). Altered smallworld brain networks in temporal lobe in patients with schizophrenia performing an auditory oddball task. Front. Syst. Neurosci. 5:7. doi:10.3389/fnsys.2011.00007

Zalesky, A., Fornito, A., Egan, G. F., Pantelis, C., and Bullmore, E. T. (2011). The relationship between regional and inter-regional functional connectivity deficits in schizophrenia. Hum. Brain Mapp. doi: 10.1002/hbm.21379. [Epub ahead of print].

Zhou, Y., Liang, M., Jiang, T., Tian, L., Liu, Y., Liu, Z., Liu, H., and Kuang, F. (2007). Functional dysconnectivity of the dorsolateral prefrontal cortex in first-episode schizophrenia using resting-state fMRI. Neurosci. Lett. 417, 297-302.

Conflict of Interest Statement: The authors declare that the research was conducted in the absence of any commercial or financial relationships that could be construed as a potential conflict of interest.

Received: 02 December 2011; accepted: 29 April 2012; published online: 23 May 2012.

Citation: Repovš G and Barch DM (2012) Working memory related brain network connectivity in individuals with schizophrenia and their siblings. Front. Hum. Neurosci. 6:137. doi: 10.3389/fnhum.2012.00137

Copyright (c) 2012 Repovš and Barch. This is an open-access article distributed under the terms of the Creative Commons Attribution Non Commercial License, which permits non-commercial use, distribution, and reproduction in other forums, provided the original authors and source are credited. 